\title{
Vibration Modeling of Bladed Disks Subject to Geometric Mistuning and Design Changes
}

\author{
Sang-Ho Lim*, Matthew P. Castanier ${ }^{\dagger}$, and Christophe Pierre ${ }^{\ddagger}$ \\ Department of Mechanical Engineering \\ The University of Michigan, Ann Arbor, MI 48109-2125
}

\begin{abstract}
In this paper, a new reduced-order modeling technique is presented for bladed disks that feature large, geometric deviations from a nominal design. Various finite-element-based reduced-order models (ROMs) have been proposed in the literature for bladed disks with small blade-to-blade differences, called mistuning. Many of these techniques rely on the fact that mistuned-system normal modes can be effectively represented using a linear combination of the normal modes of the nominal (tuned) system. However, when the mistuning or geometric deviation is large, the number of tuned-system normal modes required to describe the mistuned-system normal modes increases dramatically. In this work, a method for large mistuning is formulated by employing a mode-acceleration method with static mode compensation. By accounting for the effects of mistuning as though they were produced by external forces, a set of basis vectors is established using a combination of tuned-system normal modes compensated by static modes. The obtained basis vectors approximately span the space of the mistuned-system modes without requiring a much more expensive modal analysis of the mistuned system, and they provide much better convergence than tuned-system normal modes. Furthermore, in order to extend the method to higher frequency ranges, quasi-static modes, in which inertia effects are included, are employed in place of static modes in the modeacceleration formulation. It is seen that ROMs based on the new technique are extremely compact, yet they accurately capture the vibration response of bladed disks subject to geometric mistuning or design changes.
\end{abstract}

\section{Introduction}

For many years, researchers have investigated the vibration behavior of bladed disks. Many previous studies have been focused on the effect of small, random blade-to-blade discrepancies (mistuning). Not only is mistuning unavoidable, but the vibration response of a mistuned bladed disk can be significantly different from that of a tuned bladed disk. Although it comes from various sources, mistuning has generally been treated as a small deviation of blade mass, stiffness, or natural frequency from the nominal value in a simplified model (a lumped parameter model or a reduced-order model). ${ }^{1-15}$ However, mistuning is not necessarily small. Large, geometric variations (e.g., cracking or fracture of a blade due to fatigue or foreign object damage) can also change dramatically the dynamic behavior of a bladed disk, but these large-mistuning cases have rarely been studied.

When a lumped parameter model is employed, the system response can be easily obtained even for large mistuning. However, the number of degrees of freedom (DOF) is so small that the characteristics of an actual bladed disk may not be captured properly, especially when geometric mistuning is large. A finite element model (FEM) can predict the response of actual bladed disks realistically, regardless of the amount of mistuning. ${ }^{16}$ However, an FEM is usually computationally expensive, especially when Monte Carlo simulations are required for statistical analysis of the mistuned response. Therefore, developing a reduced-order model (ROM) of a small size is of great importance in the research of bladed disks.

Several FEM-based ROMs have been reported in recent years. ${ }^{10-15}$ However, most of these models are based on the assumption that mistuning is small. Two recently developed methods ${ }^{14,15}$ are notable for generating highly compact ROMs that feature excellent accuracy relative to the parent FEM. These models use a basis of tuned-system normal modes to capture mistuned-system normal modes. However, as mistuning becomes large, the required number

\footnotetext{
${ }^{*}$ Graduate Student Research Assistant

${ }^{\dagger}$ Associate Research Scientist, Senior Member of AIAA

${ }^{\ddagger}$ Stephen P. Timoshenko Collegiate Professor, Senior Member of AIAA
} 
of tuned-system normal modes increases dramatically, and in some cases a model gives poor results regardless of the number of retained modes.

In 1987, $\mathrm{Gu}$ and Tongue ${ }^{17}$ showed that modal convergence can be accelerated by using forced modes in addition to free vibration modes. A forced mode (or static mode) is a shape that is induced in the structure by a given external force vector. In the work by $\mathrm{Gu}$ and Tongue, external forces were applied to a beam by springs that were included as additional stiffnesses. The concept of a forced mode can be extended to a mistuned system in an analogous manner, for instance by considering mistuning as additional stiffnesses. In 1995, Cai et al. ${ }^{18}$ considered the effect of mistuning as that of external forces in a lumped parameter model, obtained a frequency equation in a closed form, and solved it. In a recent study by the authors, ${ }^{15}$ a reduced-order model for large mistuning based on a component mode synthesis (CMS) technique was proposed. In this modeling method, tuned-system normal modes (free vibration modes) and tunedsystem attachment modes (forced modes) are employed, and the ROMs show good accuracy and fast convergence with increasing number of tuned-system normal modes. However, the model size is still greater than that generated by a small-mistuning method, because one attachment-mode DOF is retained for each physical DOF in the FEM where geometric mistuning is present.

In this work, a non-CMS method for large mistuning is formulated by employing a mode-acceleration method with static mode compensation. The tuned-system normal modes are compensated by static modes that account for the effects of mistuning as though they were produced by external forces. Thus, a new set of basis vectors is established for the mistuned system. The obtained basis vectors approximately span the space of the mistuned-system modes without requiring a much more expensive modal analysis of the mistuned system, and they provide much better convergence than the original tuned-system normal modes. Furthermore, in order to extend the method to higher frequency ranges, quasi-static modes, ${ }^{19,20}$ in which inertia effects are included, are employed in place of static modes in the mode-acceleration formulation.

In addition to modeling systems with large mistuning, the presented method can also be used in the design process. Usually, when a change is made to a geometric design parameter, the new FEM must be analyzed in order to determine the effects of the design change on the system vibration response. However, if the changes to the mass and stiffness matrices due to the design change are known, the new modeling technique can be used to construct an updated ROM for the revised design without requiring additional finite element analysis of the vibration response. In this manner, the process of evaluating geometric design changes can be expedited.

This paper is organized as follows. The authors' previous approach for large mistuning is briefly reviewed in section II. In section III, the new modeling technique is formulated starting from the original mode-acceleration formulation, and it is refined using the modified mode-acceleration formulation with quasi-static modes. Then, a bladed disk with a rogue blade whose geometry deviates severely from the nominal blade design is examined as a case study in section IV. The newly developed method is validated using the parent FEM, and its performance is compared with previous methods for large and small mistuning. As further applications of this modeling technique, two additional cases are examined in sections V and VI: a bladed disk with a fractured blade, and a bladed disk subject to geometric design changes in the disk. Finally, conclusions are summarized in section VII.

\section{Background: Reduced-Order Modeling by CMS}

In this section, a general reduced-order model for large mistuning, which was developed previously by the authors, ${ }^{15}$ is briefly reviewed. A mistuned bladed disk is divided into a tuned bladed disk $\left(\boldsymbol{M}^{S}, \boldsymbol{K}^{S}\right)$ and mistuning components $\left(\boldsymbol{M}^{\delta}, \boldsymbol{K}^{\delta}\right)$ that represent the difference between the mistuned and tuned mass and stiffness matrices. The mistuned system model is constructed using a hybrid-interface CMS technique: the tuned system is treated as a free-interface component, and the mistuning components are treated as fixed-interface components. Because the mistuning components are not physically separate from the tuned system, all DOF in the mistuning components are interface DOF. Thus, both tuned-system attachment modes $\left(\boldsymbol{\Psi}^{S}\right)$ and a truncated set of normal modes $\left(\boldsymbol{\Phi}^{S}\right)$ are used to describe the displacements of the tuned system, but only constraint modes $\left(\boldsymbol{\Psi}^{\delta}=\boldsymbol{I}\right)$ are used for the mistuning components. The synthesized mass and stiffness matrices $\left(\boldsymbol{\mu}^{s y n}, \boldsymbol{\kappa}^{s y n}\right)$ of a mistuned system are assembled by enforcing displacement compatibility at the interface DOF:

$$
\begin{aligned}
\boldsymbol{\mu}^{s y n} & =\left[\begin{array}{cc}
\boldsymbol{I}+\boldsymbol{\Phi}_{\Gamma}^{S^{T}} \boldsymbol{M}^{\delta} \boldsymbol{\Phi}_{\Gamma}^{S} & \boldsymbol{\Phi}^{S^{T}} \boldsymbol{M}^{S} \boldsymbol{\Psi}^{S}+\boldsymbol{\Phi}_{\Gamma}^{S^{T}} \boldsymbol{M}^{\delta} \boldsymbol{\Psi}_{\Gamma}^{S} \\
\boldsymbol{\Psi}^{S^{T}} \boldsymbol{M}^{S} \boldsymbol{\Phi}^{S}+\boldsymbol{\Psi}_{\Gamma}^{S^{T}} \boldsymbol{M}^{\delta} \boldsymbol{\Phi}_{\Gamma}^{S} & \boldsymbol{\Psi}^{S^{T}} \boldsymbol{M}^{S} \boldsymbol{\Psi}^{S}+\boldsymbol{\Psi}_{\Gamma}^{S^{T}} \boldsymbol{M}^{\delta} \boldsymbol{\Psi}_{\Gamma}^{S}
\end{array}\right] \\
& =\left[\begin{array}{cc}
\boldsymbol{I}+\boldsymbol{\Phi}_{\Gamma}^{S^{T}} \boldsymbol{M}^{\delta} \boldsymbol{\Phi}_{\Gamma}^{S} & \boldsymbol{\Lambda}^{S^{-1}} \boldsymbol{\Phi}_{\Gamma}^{S^{T}}+\boldsymbol{\Phi}_{\Gamma}^{S^{T}} \boldsymbol{M}^{\delta} \boldsymbol{\Psi}_{\Gamma}^{S} \\
\boldsymbol{\Phi}_{\Gamma}^{S} \boldsymbol{\Lambda}^{S^{-1}}+\boldsymbol{\Psi}_{\Gamma}^{S^{T}} \boldsymbol{M}^{\delta} \boldsymbol{\Phi}_{\Gamma}^{S} & \boldsymbol{\Psi}^{S^{T}} \boldsymbol{M}^{S} \boldsymbol{\Psi}^{S}+\boldsymbol{\Psi}_{\Gamma}^{S^{T}} \boldsymbol{M}^{\delta} \boldsymbol{\Psi}_{\Gamma}^{S}
\end{array}\right]
\end{aligned}
$$




$$
\begin{aligned}
\boldsymbol{\kappa}^{s y n} & =\left[\begin{array}{cc}
\boldsymbol{\Lambda}^{S}+\boldsymbol{\Phi}_{\Gamma}^{S^{T}} \boldsymbol{K}^{\delta} \boldsymbol{\Phi}_{\Gamma}^{S} & \boldsymbol{\Phi}^{S^{T}} \boldsymbol{K}^{S} \boldsymbol{\Psi}^{S}+\boldsymbol{\Phi}_{\Gamma}^{S^{T}} \boldsymbol{K}^{\delta} \boldsymbol{\Psi}_{\Gamma}^{S} \\
\boldsymbol{\Psi}^{S^{T}} \boldsymbol{K}^{S} \boldsymbol{\Phi}^{S}+\boldsymbol{\Psi}_{\Gamma}^{S^{T}} \boldsymbol{K}^{\delta} \boldsymbol{\Phi}_{\Gamma}^{S} & \boldsymbol{\Psi}_{\Gamma}^{S}+\boldsymbol{\Psi}_{\Gamma}^{S^{T}} \boldsymbol{K}^{\delta} \boldsymbol{\Psi}_{\Gamma}^{S}
\end{array}\right] \\
& =\left[\begin{array}{ll}
\boldsymbol{\Lambda}^{S}+\boldsymbol{\Phi}_{\Gamma}^{S^{T}} \boldsymbol{K}^{\delta} \boldsymbol{\Phi}_{\Gamma}^{S} & \boldsymbol{\Phi}_{\Gamma}^{S^{T}}+\boldsymbol{\Phi}_{\Gamma}^{S^{T}} \boldsymbol{K}^{\delta} \boldsymbol{\Psi}_{\Gamma}^{S} \\
\boldsymbol{\Phi}_{\Gamma}^{S}+\boldsymbol{\Psi}_{\Gamma}^{S}{ }^{T} \boldsymbol{K}^{\delta} \boldsymbol{\Phi}_{\Gamma}^{S} & \boldsymbol{\Psi}_{\Gamma}^{S}+\boldsymbol{\Psi}_{\Gamma}^{S^{T}} \boldsymbol{K}^{\delta} \boldsymbol{\Psi}_{\Gamma}^{S}
\end{array}\right],
\end{aligned}
$$

where $\Gamma$ denotes the interface DOF where mistuning exists.

Eq. (1) shows that a mistuned system can be described with the normal modes and attachment modes of the tuned system. Since a tuned bladed disk features cyclic symmetry, normal modes and attachment modes can be obtained using only the FEM of a single sector. However, when attachment modes are involved in the CMS formulation, matrix ill-conditioning and numerical instability may occur. This is due to the fact that the displacement values of attachment modes are much smaller than those of normal modes, and also because attachment modes and normal modes may not be clearly independent. The former problem can be overcome by performing a secondary modal analysis on the attachment mode partition of the synthesized mass and stiffness matrices. In order to reduce the effect of the latter problem, the number of retained normal modes must be decreased. However, in this case, the accuracy of the ROM also decreases. To compensate for this loss of accuracy, more attachment modes can be included in the ROM. Of course, including more attachment modes leads a larger model size. In fact, in the authors' previous work, ${ }^{15}$ the size of the ROM for large mistuning using this approach was much larger than the typical size of a small-mistuning ROM. Therefore, a new, more efficient method is introduced in the next section.

\section{New Modeling Technique Using Static Mode Compensation}

In this section, a new modeling technique for a mistuned system is formulated using the mode-acceleration method. In the derivation, the effect of mistuning in a mistuned system is converted to that of external forces.

\section{A. Static Mode Compensation}

The mode-acceleration method is usually used to improve the accuracy of forced response predictions by including a static mode $\left(\boldsymbol{K}^{-1} \boldsymbol{f}\right) .{ }^{21}$ The formulation of the mode-acceleration method for an undamped system is as follows:

$$
\boldsymbol{x}=\boldsymbol{K}^{-1} \boldsymbol{f}+\sum_{i}\left(\frac{\omega^{2}}{\omega_{i}^{2}}\right) \phi_{i} \eta_{i}
$$

or

$$
\boldsymbol{x}-\boldsymbol{K}^{-1} \boldsymbol{f}=\sum_{i}\left(\frac{\omega^{2}}{\omega_{i}^{2}}\right) \boldsymbol{\phi}_{i} \eta_{i}
$$

where $\boldsymbol{x}$ is the displacement vector, $\boldsymbol{f}$ is the external force vector, $\omega$ is the excitation frequency, $\omega_{i}$ is the natural frequency of the $i$ th mode, $\phi_{i}$ is the $i$ th normal mode, and $\eta_{i}$ is the $i$ th modal amplitude that is used in the modedisplacement method. In Eq. (3), $\boldsymbol{x}-\boldsymbol{K}^{-1} \boldsymbol{f}$ is expressed as a linear combination of normal modes. Note that, in this combination, lower normal modes will dominate due to the coefficients, $\omega^{2} / \omega_{i}^{2}$, if the external forces excite every

mode evenly. That is, $\boldsymbol{x}-\boldsymbol{K}^{-1} \boldsymbol{f}$ can be described with a small set of normal modes. Now, suppose that the normal modes are unknown, but a set of vectors of the form $\boldsymbol{x}-\boldsymbol{K}^{-1} \boldsymbol{f}$ are known in a lower frequency range. Then, reversely, lower normal modes can be obtained accurately by employing the set of $\boldsymbol{x}-\boldsymbol{K}^{-1} \boldsymbol{f}$ vectors as a basis, unless $\boldsymbol{f}$ excites higher modes much more than lower modes.

This concept can be applied to a mistuned system when the tuned-system normal modes are known. Consider a forced response case in which a mistuned system is vibrating at a natural frequency of a tuned-system mode and the motion of the mistuned system is exactly the same as that of the tuned-system mode. Then, Eq. (3) can be rewritten as:

$$
\phi_{j}^{S}-\boldsymbol{K}^{m-1} \boldsymbol{f}_{j}=\sum_{i}\left(\frac{\omega_{j}^{S^{2}}}{\omega_{i}^{m 2}}\right) \boldsymbol{\phi}_{i}^{m} \eta_{i j} .
$$

The external forces required to enforce this motion are

$$
\boldsymbol{f}_{j}=\left[-\omega_{j}^{S^{2}} \boldsymbol{M}^{m}+\boldsymbol{K}^{m}\right] \boldsymbol{\phi}_{j}^{S}=\left\{\begin{array}{c}
\mathbf{0} \\
{\left[-\omega_{j}^{S^{2}} \boldsymbol{M}^{\delta}+\boldsymbol{K}^{\delta}\right] \boldsymbol{\phi}_{\Gamma, j}^{S}}
\end{array}\right\},
$$


where $\boldsymbol{M}^{m}$ and $\boldsymbol{K}^{m}$ are the mass and stiffness matrices of a mistuned system, $\omega_{j}^{S}$ and $\boldsymbol{\phi}_{j}^{S}$ are the $j$ th natural frequency and mode shape of the tuned system, and $\eta_{i j}$ is the modal participation factor of the $i$ th mistuned-system normal mode for the $j$ th tuned-system normal mode. The advantage of using tuned modes is that tuned-system modes are readily available because an analysis of a bladed disk usually starts with computing tuned modes that can be obtained from the FEM of a single sector. Another advantage is that non-zero forcing terms appear only at the DOF where mistuning exists, as indicated by the partitioning of the right-hand side of Eq. (5).

The static modes $\boldsymbol{K}^{m-1} \boldsymbol{f}_{j}$ can be obtained from the FEM of the mistuned system. It is also possible to obtain the static modes from the FEM of the tuned system by using the following relation:

$$
\boldsymbol{K}^{m-1} \boldsymbol{f}_{j}=\boldsymbol{K}^{S^{-1}}\left(\boldsymbol{I}+\overline{\boldsymbol{K}}^{\delta} \boldsymbol{K}^{S^{-1}}\right)^{-1} \boldsymbol{f}_{j}=\boldsymbol{K}^{S^{-1}} \boldsymbol{g}_{j},
$$

where

$$
\boldsymbol{g}_{j}=\left(\boldsymbol{I}+\overline{\boldsymbol{K}}^{\delta} \boldsymbol{K}^{S^{-1}}\right)^{-1} \boldsymbol{f}_{j}=\left\{\begin{array}{c}
\mathbf{0} \\
\left(\boldsymbol{I}+\boldsymbol{K}^{\delta} \boldsymbol{\Psi}_{\Gamma}^{S}\right)^{-1} \boldsymbol{f}_{\Gamma, j}
\end{array}\right\},
$$

$\boldsymbol{K}^{S}$ denotes stiffness matrix of a tuned system, $\overline{\boldsymbol{K}}^{\delta}$ denotes a matrix of the same size as that of $\boldsymbol{K}^{S}$, which consists of $\boldsymbol{K}^{\delta}$ and zero terms, and $\boldsymbol{\Psi}^{S}$ is a set of tuned-system attachment modes. That is, the static deflection of a mistuned system induced by the forces $\boldsymbol{f}_{j}$ is the static deflection of a tuned system induced by the forces $\boldsymbol{g}_{j}$. Here, since $\boldsymbol{f}_{j}$ has non-zero terms for only the DOF where mistuning exists, so does $\boldsymbol{g}_{j}$. Computing static modes of a mistuned system by using a tuned system is especially useful for a bladed disk system, because any static deflection of a tuned bladed disk can be computed using only the FEM of a single sector.

Static modes can be obtained by directly applying the forces, $\boldsymbol{f}_{j}$ or $\boldsymbol{g}_{j}$, or they can be computed as a linear combination of tuned-system attachment modes with the coefficients being the corresponding forces. That is,

$$
\boldsymbol{K}^{m-1} \boldsymbol{f}_{j}=\boldsymbol{K}^{S^{-1}} \boldsymbol{g}_{j}=\boldsymbol{\Psi}^{S} \boldsymbol{g}_{\Gamma, j} .
$$

If many mistuned systems need to be analyzed, the latter method would be more efficient, because the obtained attachment modes can be used for any $\boldsymbol{K}^{\delta}$. However, if the number of mistuning DOF is so large that the computation of $\boldsymbol{\Psi}^{S}$ and $\boldsymbol{g}_{\Gamma, j}$ requires higher cost, one should consider computing $\boldsymbol{K}^{m-1} \boldsymbol{f}_{j}$ or $\boldsymbol{K}^{S^{-1}} \boldsymbol{g}_{j}$ directly.

Now, a truncated set of tuned-system normal modes compensated by static modes, $\phi_{j}^{S}-\boldsymbol{K}^{S^{-1}} \boldsymbol{g}_{j}$, may be used as an alternative basis that approximately spans the space of the lower mistuned-system normal modes. The reduced mass and stiffness matrices are:

$$
\begin{aligned}
\boldsymbol{\mu}^{s y n}= & \left(\boldsymbol{\Phi}^{S}-\boldsymbol{\Psi}^{S} \boldsymbol{G}_{\Gamma}\right)^{T}\left(\boldsymbol{M}^{S}+\boldsymbol{M}^{\delta}\right)\left(\boldsymbol{\Phi}^{S}-\boldsymbol{\Psi}^{S} \boldsymbol{G}_{\Gamma}\right) \\
= & \boldsymbol{I}+\boldsymbol{\Phi}_{\Gamma}^{S^{T}} \boldsymbol{M}^{\delta} \boldsymbol{\Phi}_{\Gamma}^{S}-\left(\boldsymbol{\Lambda}^{S^{-1}} \boldsymbol{\Phi}_{\Gamma}^{S^{T}}+\boldsymbol{\Phi}_{\Gamma}^{S^{T}} \boldsymbol{M}^{\delta} \boldsymbol{\Psi}_{\Gamma}^{S}\right) \boldsymbol{G}_{\Gamma} \\
& -\boldsymbol{G}_{\Gamma}^{T}\left(\boldsymbol{\Phi}_{\Gamma}^{S} \boldsymbol{\Lambda}^{S^{-1}}+\boldsymbol{\Psi}_{\Gamma}^{S^{T}} \boldsymbol{M}^{\delta} \boldsymbol{\Phi}_{\Gamma}^{S}\right)+\boldsymbol{G}_{\Gamma}^{T}\left(\boldsymbol{\Psi}^{S^{T}} \boldsymbol{M}^{S} \boldsymbol{\Psi}^{S}+\boldsymbol{\Psi}_{\Gamma}^{S^{T}} \boldsymbol{M}^{\delta} \boldsymbol{\Psi}_{\Gamma}^{S}\right) \boldsymbol{G}_{\Gamma} \\
\boldsymbol{\kappa}^{s y n}= & \left(\boldsymbol{\Phi}^{S}-\boldsymbol{\Psi}^{S} \boldsymbol{G}_{\Gamma}\right)^{T}\left(\boldsymbol{K}^{S}+\boldsymbol{K}^{\delta}\right)\left(\boldsymbol{\Phi}^{S}-\boldsymbol{\Psi}^{S} \boldsymbol{G}_{\Gamma}\right) \\
= & \boldsymbol{\Lambda}^{S}+\boldsymbol{\Phi}_{\Gamma}^{S^{T}} \boldsymbol{K}^{\delta} \boldsymbol{\Phi}_{\Gamma}^{S}-\left(\boldsymbol{\Phi}_{\Gamma}^{S^{T}}+\boldsymbol{\Phi}_{\Gamma}^{S^{T}} \boldsymbol{K}^{\delta} \boldsymbol{\Psi}_{\Gamma}^{S}\right) \boldsymbol{G}_{\Gamma} \\
& -\boldsymbol{G}_{\Gamma}^{T}\left(\boldsymbol{\Phi}_{\Gamma}^{S}+\boldsymbol{\Psi}_{\Gamma}^{S^{T}} \boldsymbol{K}^{\delta} \boldsymbol{\Phi}_{\Gamma}^{S}\right)+\boldsymbol{G}_{\Gamma}^{T}\left(\boldsymbol{\Psi}_{\Gamma}^{S}+\boldsymbol{\Psi}_{\Gamma}^{S^{T}} \boldsymbol{K}^{\delta} \boldsymbol{\Psi}_{\Gamma}^{S}\right) \boldsymbol{G}_{\Gamma},
\end{aligned}
$$

where the matrix $\boldsymbol{G}_{\Gamma}$ is a set of the vectors $\boldsymbol{g}_{\Gamma, j}$. The size of these reduced matrices is $N \times N$, where $N$ is the number of tuned-system normal modes in a truncated set. As mentioned above, the accuracy will be determined by $\left(\omega_{j}^{S^{2}} / \omega_{i}^{2}\right) \eta_{i j}$. If the value of $\left(\omega_{j}^{S^{2}} / \omega_{i}^{2}\right) \eta_{i j}$ for the $i$ th mode is relatively small compared to those for other modes, the $i$ th mode obtained by this method will be less accurate. That is, mistuned-system normal modes in a high frequency range may be inaccurate because the value of $\omega_{j}^{S^{2}} / \omega_{i}^{2}$ is smaller for a high mode than for a low mode.

\section{B. Quasi-Static Mode Compensation}

For a better representation in a higher frequency range, the formulation of the mode-acceleration method in Eq. (2) is modified. First, the equations of motion of an undamped system are written as follows:

$$
\left[-\omega^{2} \boldsymbol{M}+\boldsymbol{K}\right] \boldsymbol{x}=\left[-\left(\omega^{2}-\omega_{c}^{2}\right) \boldsymbol{M}+\left(\boldsymbol{K}-\omega_{c}^{2} \boldsymbol{M}\right)\right] \boldsymbol{x}=\boldsymbol{f}
$$


where $\omega_{c}$ is a pre-determined frequency, which is the centering frequency used in the quasi-static mode compensation method introduced by Shyu et al. ${ }^{19,20}$ to improve CMS models in higher frequency ranges. As can be seen in Eq. (10), inertial effects corresponding to a centering frequency are transfered to the stiffness term. Thereby, the motion of the original system, $\boldsymbol{M}$ and $\boldsymbol{K}$, at a frequency of $\omega$ becomes the same as the motion of an equivalent system, $M$ and $\boldsymbol{K}-\omega_{c}^{2} \boldsymbol{M}$, at a frequency of $\sqrt{\omega^{2}-\omega_{c}^{2}}$. The equivalent system has the same mode shapes as those of the original system. However, its eigenvalues are shifted by $-\omega_{c}^{2}$ from the original values. Note that the original system can be considered as a special case in which the centering frequency is zero.

Applying the mode-acceleration method to this equivalent system, $\boldsymbol{x}$ becomes

$$
\begin{aligned}
\boldsymbol{x} & =\left(\boldsymbol{K}-\omega_{c}^{2} \boldsymbol{M}\right)^{-1} \boldsymbol{f}+\left(\boldsymbol{K}-\omega_{c}^{2} \boldsymbol{M}\right)^{-1}\left(\omega^{2}-\omega_{c}^{2}\right) \boldsymbol{M} \boldsymbol{x} \\
& =\left(\boldsymbol{K}-\omega_{c}^{2} \boldsymbol{M}\right)^{-1} \boldsymbol{f}+\sum_{i}\left(\frac{\omega^{2}-\omega_{c}^{2}}{\omega_{i}^{2}-\omega_{c}^{2}}\right) \boldsymbol{\phi}_{i} \eta_{i} .
\end{aligned}
$$

where $\left(\boldsymbol{K}-\omega_{c}^{2} \boldsymbol{M}\right)^{-1} \boldsymbol{f}$ are quasi-static modes. ${ }^{19,20}$ Now, as can be seen in Eq. (11), the coefficient of $\boldsymbol{\phi}_{i}$ is $\left[\left(\omega^{2}-\omega_{c}^{2}\right) /\left(\omega_{i}^{2}-\omega_{c}^{2}\right)\right] \eta_{i}$. Therefore, if $\omega_{i}$ is close to $\omega_{c}$, the coefficient of the $i$ th mode can have a larger value than the other modes. Therefore, $\boldsymbol{x}-\left(\boldsymbol{K}-\omega_{c}^{2} \boldsymbol{M}\right)^{-1} \boldsymbol{f}$ can be described by a small number of mistuned modes around the centering frequency.

Following the same procedure as that for static mode compensation using the original mode-acceleration formulation, the quasi-static modes of a mistuned system can be computed from the tuned system as follows:

$$
\left(\boldsymbol{K}^{m}-\omega_{c}^{2} \boldsymbol{M}^{m}\right)^{-1} \boldsymbol{f}_{j}=\boldsymbol{\Psi}^{S, Q} \boldsymbol{p}_{\Gamma, j},
$$

where

$$
\boldsymbol{p}_{\Gamma, j}=\left[\boldsymbol{I}+\left(\boldsymbol{K}^{\delta}-\omega_{c}^{2} \boldsymbol{M}^{\delta}\right) \boldsymbol{\Psi}^{S, Q}\right] \boldsymbol{f}_{\Gamma, j}
$$

and $\boldsymbol{\Psi}^{S, Q}$ is a set of quasi-static attachment modes computed from the equivalent stiffness matrix $\boldsymbol{K}^{S}-\omega_{c}^{2} \boldsymbol{M}^{S}$. Now, the reduced mass and stiffness matrices are

$$
\begin{aligned}
\boldsymbol{\mu}^{s y n}= & \left(\boldsymbol{\Phi}^{S}-\boldsymbol{\Psi}^{S, Q} \boldsymbol{P}_{\Gamma}\right)^{T}\left(\boldsymbol{M}^{S}+\boldsymbol{M}^{\delta}\right)\left(\boldsymbol{\Phi}^{S}-\boldsymbol{\Psi}^{S, Q} \boldsymbol{P}_{\Gamma}\right) \\
= & \boldsymbol{I}+\boldsymbol{\Phi}_{\Gamma}^{S^{T}} \boldsymbol{M}^{\delta} \boldsymbol{\Phi}_{\Gamma}^{S}-\left[\left(\boldsymbol{\Lambda}^{S}-\omega_{c}^{2} \boldsymbol{I}\right)^{-1} \boldsymbol{\Phi}_{\Gamma}^{S^{T}}+\boldsymbol{\Phi}_{\Gamma}^{S^{T}} \boldsymbol{M}^{\delta} \boldsymbol{\Psi}_{\Gamma}^{S, Q}\right] \boldsymbol{P}_{\Gamma} \\
& -\boldsymbol{P}_{\Gamma}^{T}\left[\boldsymbol{\Phi}_{\Gamma}^{S}\left(\boldsymbol{\Lambda}^{S}-\omega_{c}^{2} \boldsymbol{I}\right)^{-1}+\boldsymbol{\Psi}_{\Gamma}^{S, Q^{T}} \boldsymbol{M}^{\delta} \boldsymbol{\Phi}_{\Gamma}^{S}\right] \\
& +\boldsymbol{P}_{\Gamma}^{T}\left(\boldsymbol{\Psi}^{S, Q^{T}} \boldsymbol{M}^{S} \boldsymbol{\Psi}^{S, Q}+\boldsymbol{\Psi}_{\Gamma}^{S, Q^{T}} \boldsymbol{M}^{\delta} \boldsymbol{\Psi}_{\Gamma}^{S, Q}\right) \boldsymbol{P}_{\Gamma} \\
\boldsymbol{\kappa}^{s y n}= & \left(\boldsymbol{\Phi}^{S}-\boldsymbol{\Psi}^{S, Q} \boldsymbol{P}_{\Gamma}\right)^{T}\left(\boldsymbol{K}^{S}+\boldsymbol{K}^{\delta}\right)\left(\boldsymbol{\Phi}^{S}-\boldsymbol{\Psi}^{S, Q} \boldsymbol{P}_{\Gamma}\right) \\
= & \boldsymbol{\Lambda}^{S}+\boldsymbol{\Phi}_{\Gamma}^{S^{T}} \boldsymbol{K}^{\delta} \boldsymbol{\Phi}_{\Gamma}^{S}-\left[\boldsymbol{\Lambda}^{S}\left(\boldsymbol{\Lambda}^{S}-\omega_{c}^{2} \boldsymbol{I}\right)^{-1} \boldsymbol{\Phi}_{\Gamma}^{S^{T}}+\boldsymbol{\Phi}_{\Gamma}^{S^{T}} \boldsymbol{K}^{\delta} \boldsymbol{\Psi}_{\Gamma}^{S, Q}\right] \boldsymbol{P}_{\Gamma} \\
& -\boldsymbol{P}_{\Gamma}^{T}\left[\boldsymbol{\Phi}_{\Gamma}^{S}\left(\boldsymbol{\Lambda}^{S}-\omega_{c}^{2} \boldsymbol{I}\right)^{-1} \boldsymbol{\Lambda}^{S}+\boldsymbol{\Psi}_{\Gamma}^{S, Q^{T}} \boldsymbol{K}^{\delta} \boldsymbol{\Phi}_{\Gamma}^{S}\right] \\
& +\boldsymbol{P}_{\Gamma}^{T}\left(\boldsymbol{\Psi}_{\Gamma}^{S, Q}+\omega_{c}^{2} \boldsymbol{\Psi}^{S, Q^{T}} \boldsymbol{M}^{S} \boldsymbol{\Psi}^{S, Q}+\boldsymbol{\Psi}_{\Gamma}^{S, Q^{T}} \boldsymbol{K}^{\delta} \boldsymbol{\Psi}_{\Gamma}^{S, Q}\right) \boldsymbol{P}_{\Gamma},
\end{aligned}
$$

where $\boldsymbol{P}_{\Gamma}$ is a set of $\boldsymbol{p}_{\Gamma, j}$.

The accuracy of this approach depends primarily on the value of $\left[\left(\omega_{j}^{S^{2}}-\omega_{c}^{2}\right) /\left(\omega_{i}^{2}-\omega_{c}^{2}\right)\right] \eta_{i j}$. If $\omega_{j}^{S}=\omega_{c}$, then $\phi_{j}^{S}-\boldsymbol{\Psi}^{S, Q} \boldsymbol{p}_{\Gamma, j}$ will be a null vector, and the reduced mass and stiffness matrices will have a null column and a null row. If $\omega_{i}=\omega_{c}$, then the inverse of $\boldsymbol{K}^{m}-\omega_{c}^{2} \boldsymbol{M}^{m}$ will not exist. So, $\omega_{c}$ should be chosen so that it is not too close to a natural frequency of the tuned or mistuned system.

Note that it is possible to introduce an iteration scheme to the method presented in this section, or to use a higher order expression for the mode-acceleration method in order to improve accuracy. However, in those cases, the external force vectors $(\boldsymbol{G}$ or $\boldsymbol{P})$ used for the computation of static modes would be fully populated, and thus the whole set of attachment modes would be needed, and the inversion of a full-system size of matrix would be required. Thus, the cost of such a scheme would probably be prohibitive. 


\section{Comparison of Methods}

In this section, the newly developed static mode compensation (SMC) technique is validated by examining the vibration response of a turbine engine compressor stage with a rogue blade that has a significant geometric distortion relative to the nominal blade design. Also, the performance of the SMC method is compared with three other methods:

1. The CMS method for large mistuning, ${ }^{15}$ which was reviewed in section II.

2. Classical modal analysis (CMA) for small mistuning. In this model, a subset of tuned-system normal modes are employed, ${ }^{14}$ and blade mistuning is projected directly onto the system modes.

3. Component mode mistuning (CMM) technique ${ }^{15}$ for small mistuning. As in the above model, tuned-system normal modes are used for building a ROM. However, mistuning is projected to tuned-system normal modes by relating the blade motion in the system modes to the tuned cantilevered-blade normal modes. Thereby, eigenvalue mistuning of a cantilevered blade is projected onto the system modes.

The second and third techniques listed above are extremely accurate and efficient, relative to finite element analysis, for small-mistuning cases. ${ }^{14,15}$

For the SMC technique and the two small-mistuning methods, the model size is determined by the number of tuned-system normal modes selected. However, the model size for the CMS-based large-mistuning method is much larger, because the number of DOF is the number of tuned-system normal modes plus the number of attachment modes employed. For this study, the tuned-system normal modes and attachment modes were obtained from the single-sector FEM. Also, the static deflections of a tuned system due to external forces were obtained from this FEM. For the testcase rotor, the number of DOF where mistuning exists due to the geometry deviation is 594, and thus 594 attachment modes are used.

\section{A. Description of the Test-Case Model}

The rotor considered in this study is a 29-blade compressor stage of a gas turbine engine that was used in a previous study by the authors. ${ }^{15}$ Figure 1 shows the finite element mesh of this rotor, which is constructed with standard linear brick elements (eight-noded solids) and has 126,846 DOF. This figure also shows a tuned blade of the nominal design. For this test case, the effect of having one damaged blade with significant geometric mistuning, or a rogue blade, is investigated. The rogue blade geometry used in this study is shown in Fig. 2. The geometry corresponding to the worst-case blade damage is referred to as $100 \%$ distortion. By scaling the difference between the nominal and $100 \%$ distortion models, intermediate cases were generated, such as the 10\% distortion case shown in Fig. 2. The Young's modulus and mass density values used for the rogue blade were the same as those for the nominal blade. Nevertheless, due to the geometry change, the mass and stiffness matrices were significantly changed around the distorted geometry, even for the case of $10 \%$ distortion.

First, the natural frequencies and mode shapes of the rogue blade were investigated with increasing distortion. Figure 3a) shows geometry distortion versus eigenvalue mistuning for the 1st (first flexural mode, FEM natural frequency $2.22 \mathrm{kHz}$ ), 5th (second torsion, $20.95 \mathrm{kHz}$ ), 8th (third torsion, $33.68 \mathrm{kHz}$ ), and 9th (second stripe, 35.11 $\mathrm{kHz}$ ) modes of a cantilevered blade that was fixed at its root. An eigenvalue mistuning value is the ratio of the eigenvalue deviation to the nominal eigenvalue. The modal assurance criterion (MAC) values between the modes of a nominal blade and a rogue blade are shown in Fig. 3b). Although all the eigenvalue mistuning values are smaller than 0.07 , it is seen that the mode shapes are significantly different. The mode shapes of cantilevered tuned and rogue (100\% distortion) blades are depicted in Fig. 4. As can be seen, the 1st and 5th modes of a tuned and a rogue blades are similar, while the 8th and 9th modes are quite different.

The tuned system of the test-case model has many mode groups that can be characterized by blade motion (see Fig. 2 in the paper by Lim et al. ${ }^{15}$ ). Similarly, the modes of the mistuned system can be characterized by blade motion, but cannot be characterized by the number of nodal diameters. In Fig. 5, the natural frequencies for the tuned system and for the mistuned system with a rogue blade (100\% distortion) are shown for the 1st, 5th, 8th, and 9th blade-dominated mode groups. The blade motion for each system mode is highly correlated with that of a tuned or rogue cantilevered blade. Note that each mode group has one mistuned-system mode whose natural frequency is away from the others: the mode at $2.1405 \mathrm{kHz}$ in the 1 st group, the mode at $19.605 \mathrm{kHz}$ in the 5 th group, the mode at 32.912 $\mathrm{kHz}$ in the 8th group, and the mode at $34.350 \mathrm{kHz}$ in the 9th group. These modes are localized about the rogue blade, as shown in Fig. 5. Since these modes are extremely localized, it is expected that the effect of these modes on the forced responses will appear for any engine order of excitation. Therefore, the ability to capture these modes is an important consideration for assessing the performance of a test-case ROM. 


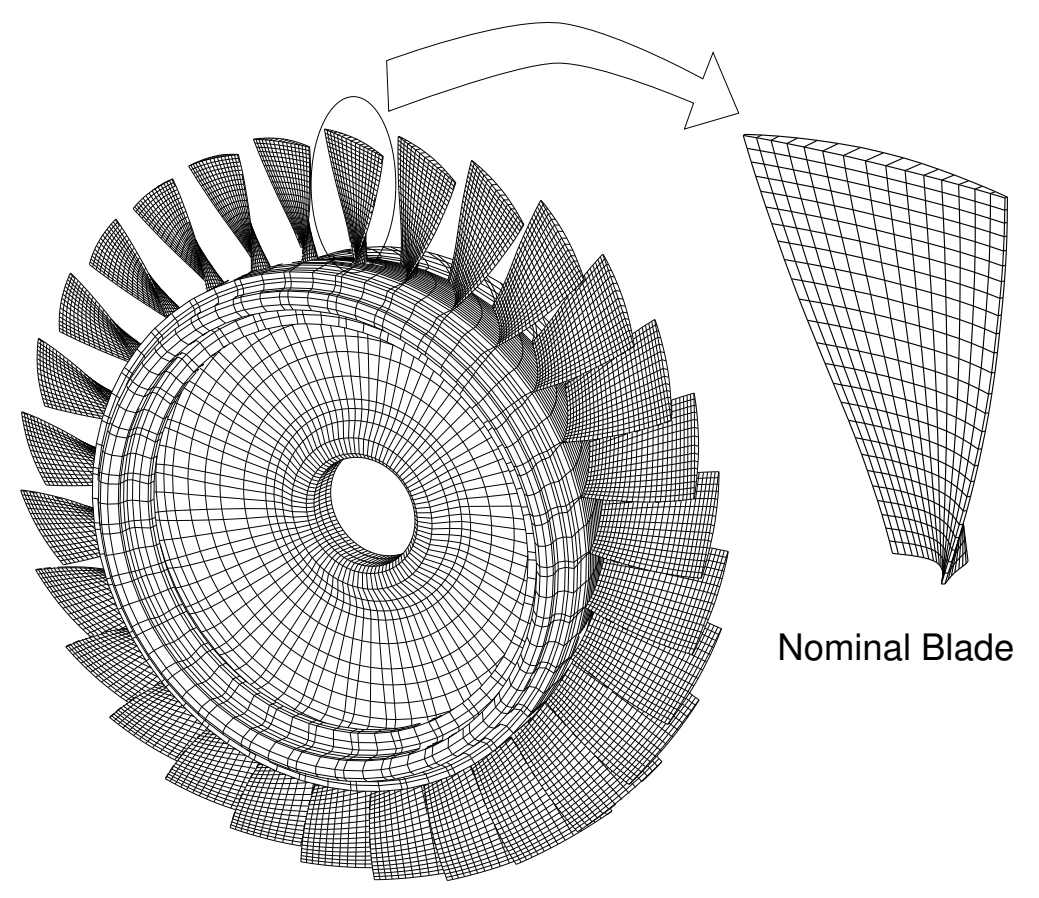

Figure 1: Finite element mesh of the test case rotor

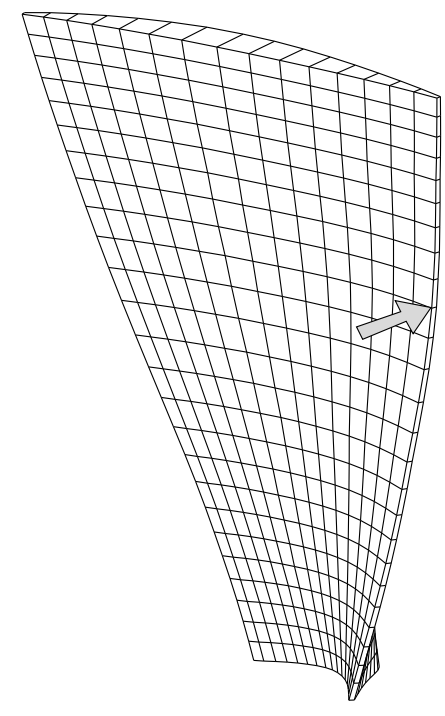

10\% Distortion

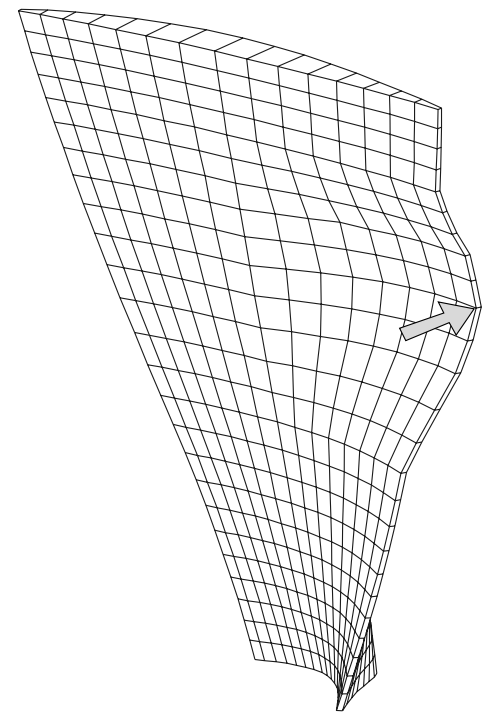

100\% Distortion

Figure 2: Rogue blade geometry 


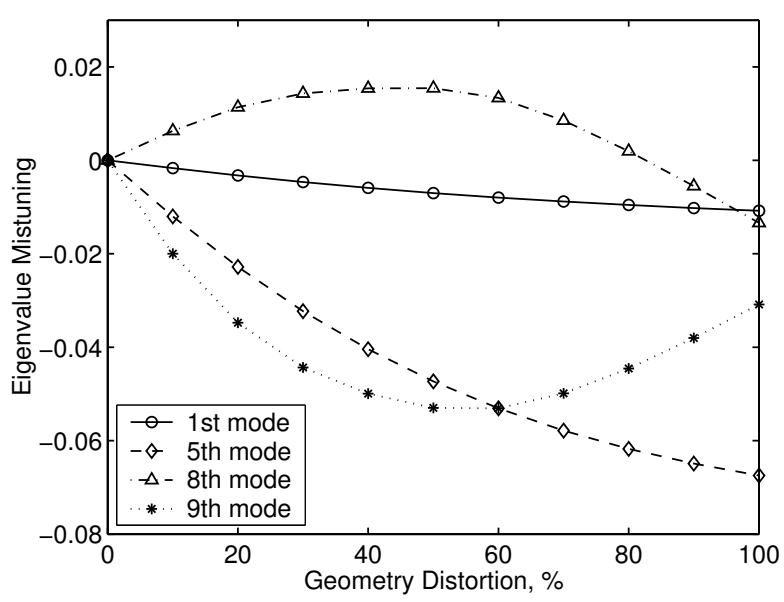

a) Eigenvalue mistuning of a cantilevered rogue blade

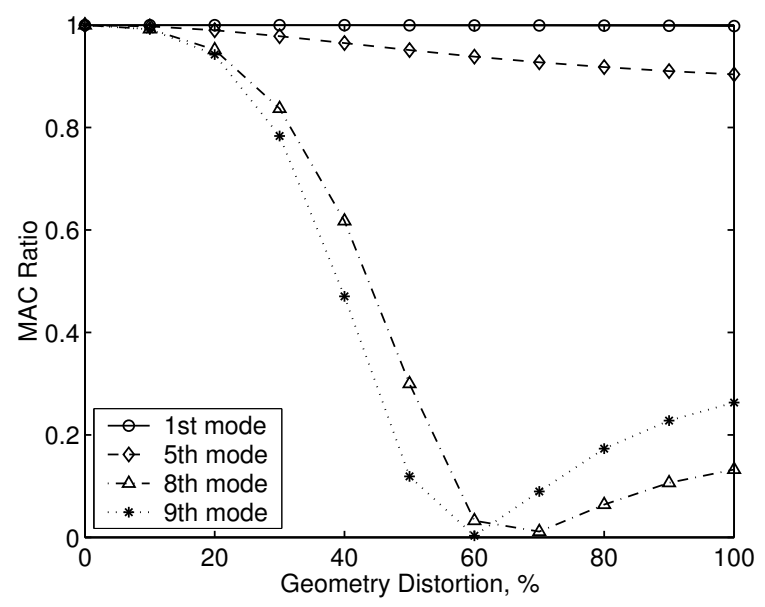

b) MAC values between cantilevered tuned and rogue blades

Figure 3: Effect of increasing rogue blade geometry distortion on free response

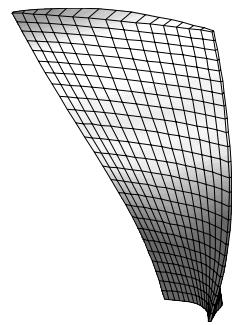

1st tuned mode

$(2221.6 \mathrm{~Hz})$

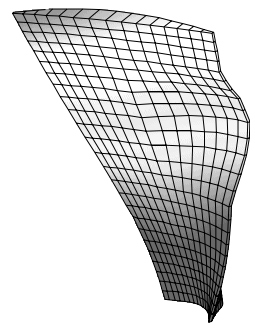

1st rogue mode $(2209.6 \mathrm{~Hz})$

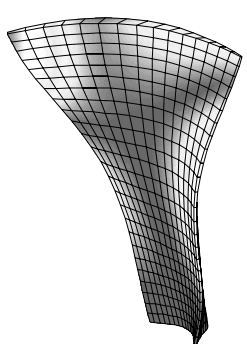

5th tuned mode $(20954.2 \mathrm{~Hz})$

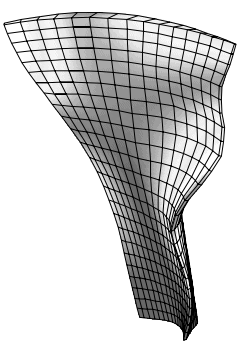

5th rogue mode $(20235.1 \mathrm{~Hz})$

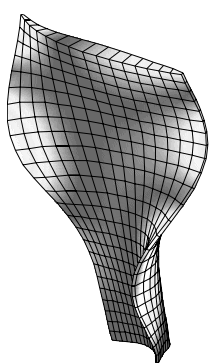

8 th tuned mode $(33679.5 \mathrm{~Hz})$

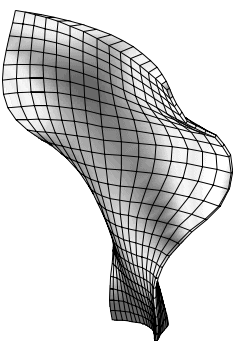

8th rogue mode $(33453.3 \mathrm{~Hz})$

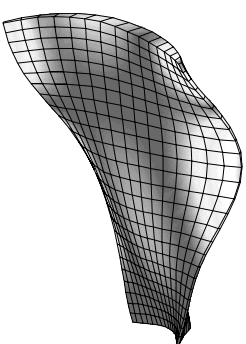

9th tuned mode $(35108.4 \mathrm{~Hz})$

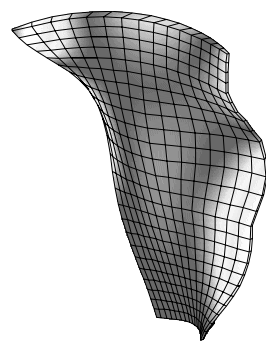

9th rogue mode $(34562.6 \mathrm{~Hz})$

Figure 4: Mode shapes of a tuned blade and a rogue blade (100\% distortion) 

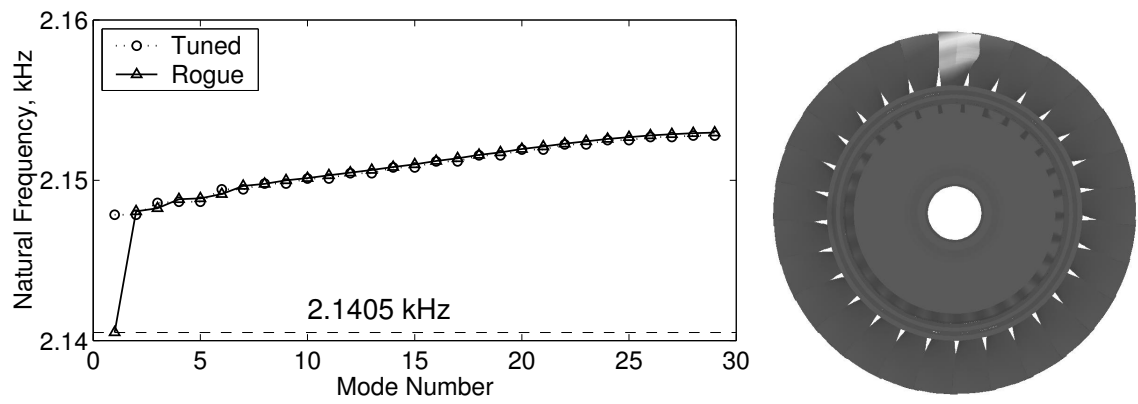

a) Natural frequencies in the 1st mode group, rogue-blade-dominated mode at $2.1405 \mathrm{kHz}$
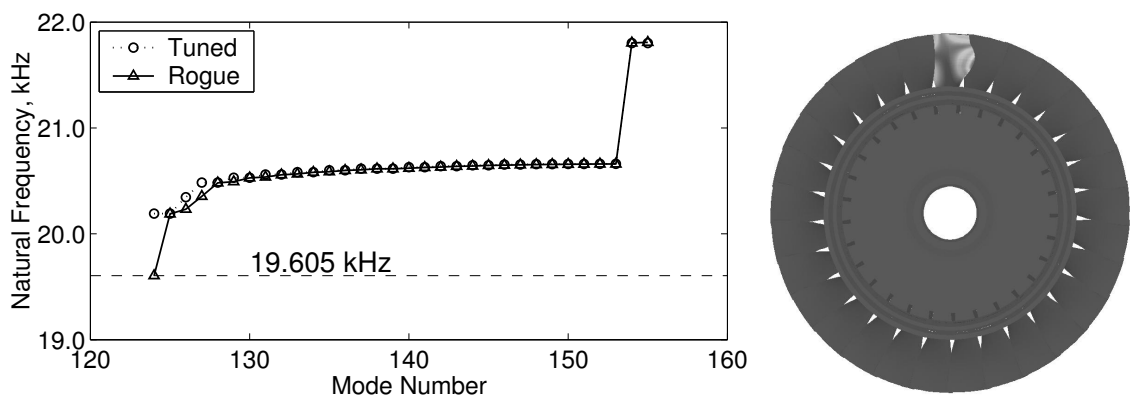

b) Natural frequencies in the 5th mode group, rogue-blade-dominated mode at $19.605 \mathrm{kHz}$
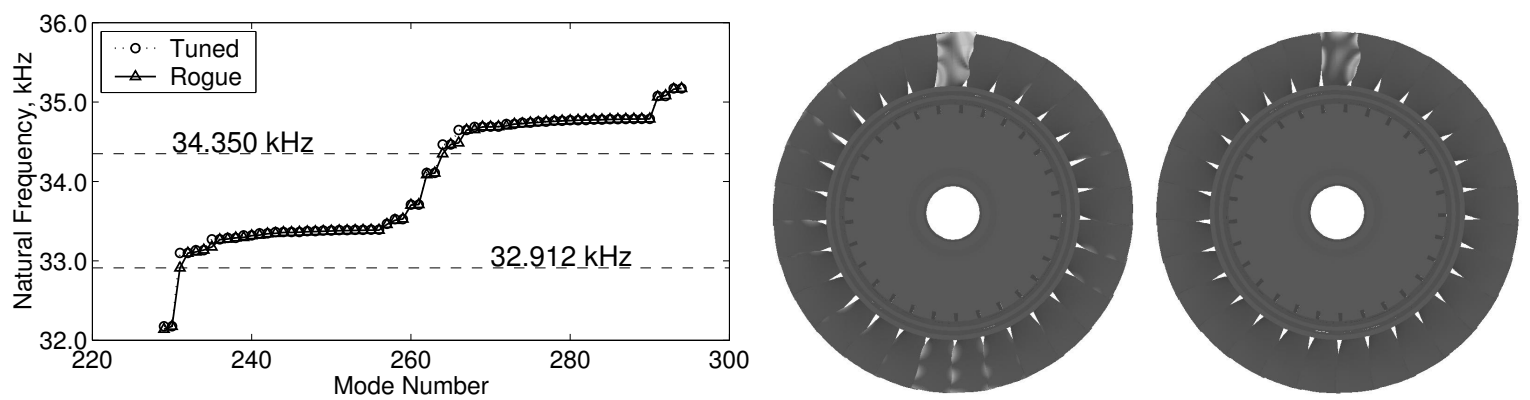

c) Natural frequencies in the 8th and 9th mode groups, rogue-blade-dominated modes at $32.912 \mathrm{kHz}$ and $34.350 \mathrm{kHz}$

Figure 5: Natural frequencies and mode shapes of a bladed disk with a rogue blade (100\% distortion) 


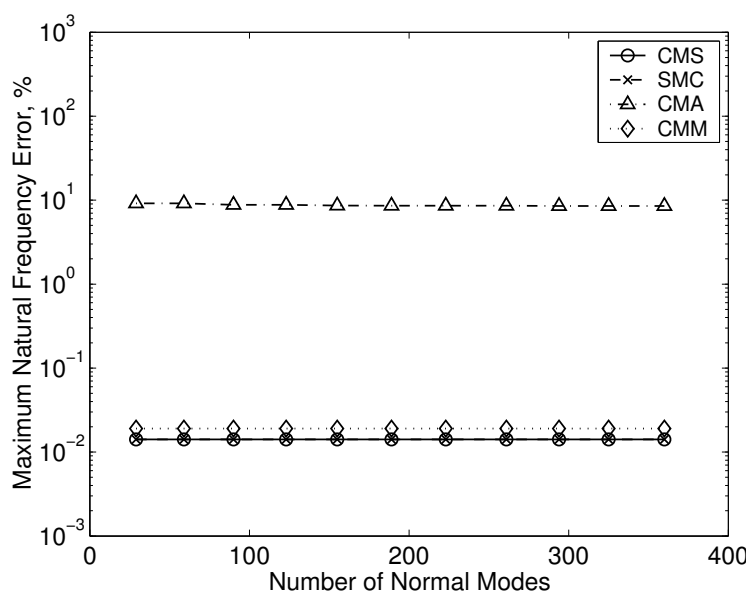

a) $10 \%$ distortion

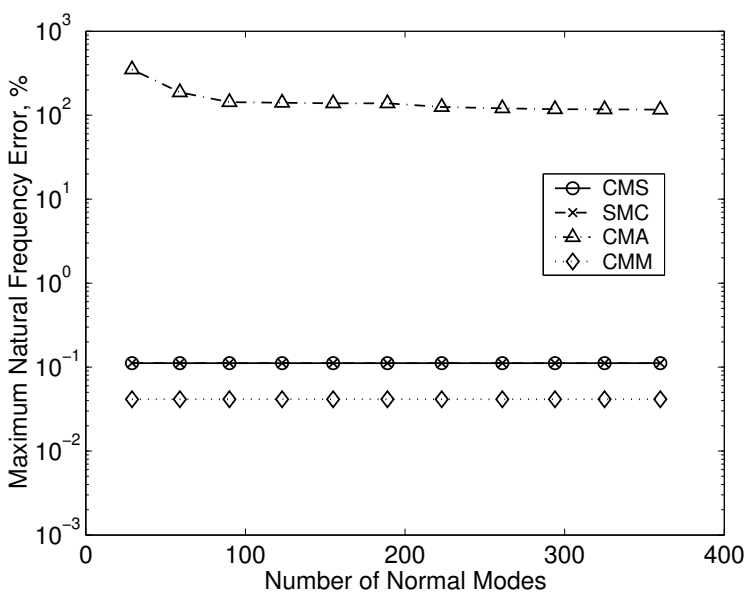

b) $100 \%$ distortion

Figure 6: Convergence of natural frequency errors for the 1st blade-dominated mode group

\section{B. Free Response Results}

Figures 6 and 7 present the convergence to the FEM results of ROM natural frequencies for the four different methods: CMS (Component Mode Synthesis, o), SMC (Static Mode Compensation using the mode-acceleration formulation, $\times$ ), CMA (Classical Modal Analysis with mistuning projection, $\triangle$ ), and CMM (Component Mode Mistuning, $\diamond$ ). The rogue-blade-dominated modes mentioned in the previous section had much larger error than the others. Therefore, maximum frequency errors were used for the convergence study.

Figure 6 shows the maximum natural frequency errors for the lowest blade-dominant mode group as the number of tuned-system normal modes increases, for the cases of $10 \%$ distortion and 100\% distortion. There are 29 mistuned normal modes in the lowest mode group. For the SMC method, static, not quasi-static, modes are used, because the modes of interest are the lowest modes. For the 1st cantilevered blade mode, the mode shapes of tuned and rogue blades were almost the same (the MAC value at 100\% deviation is 0.9982). However, as can be seen in Fig. 6, the results from the CMA method are poor, even for the case of $10 \%$ geometry distortion.

Figure 7 shows natural frequency errors of the 5th and the 8th-9th blade-dominated mode groups for the case of a rogue blade with $100 \%$ deviation. There are 32 normal modes in the 5th mode group, and 66 normal modes in the 8th and 9th mode groups. The 8th and 9th mode groups are so close that they need to be included in a single ROM. The results by the CMA method were excluded because the errors were too large. The models by the SMC method were obtained using quasi-static modes. The used centering frequency was $20 \mathrm{kHz}$ for the 5 th mode group, and $34 \mathrm{kHz}$ for the 8th and 9th mode groups. For fair comparison, the models by the CMS method were constructed using quasi-static attachment modes, and the number of normal modes was increased by including both higher and lower modes around the centering frequencies. As shown in Fig. 7, the SMC method gives the best results. The CMS method also shows good results after many more normal modes are retained. The results by the CMS method in this paper are different from those in the authors' previous paper. ${ }^{15}$ This is because 594 attachment modes were used in this study, while 2496 attachment modes were used in the previous work. The maximum errors for the 8th and 9th mode groups by the CMM method are around $0.4 \%$, which is still very small. However, the ratio of the standard deviation of the natural frequencies to the average natural frequency is $2.3 \%$ for the 8 th and 9 th mode groups. So, an error of $0.4 \%$ may not be acceptable.

Figure 8 illustrates a tuned-system normal mode, a static mode, a quasi-static mode, and the resulting basis shapes used in the SMC method. All the displacements of these modes and shapes were applied on the geometry of the bladed disk with a rogue blade. As can be seen in this figure, the basis shapes obtained by a static mode and by a quasi-static mode are very different. In addition, the motion of a rogue blade in the basis shape obtained by a quasi-static mode is very similar to the 9th cantilevered-blade mode of a rogue blade shown in Fig. 4. Therefore, it is clear that using quasi-static modes can significantly improve the convergence of a model in a higher frequency region.

The rogue-blade dominated mode in each mode group was selected for the test of the accuracy of mode shape representation. Table 1 shows the convergence of the MAC values between the modes by ROMs and the modes by the 


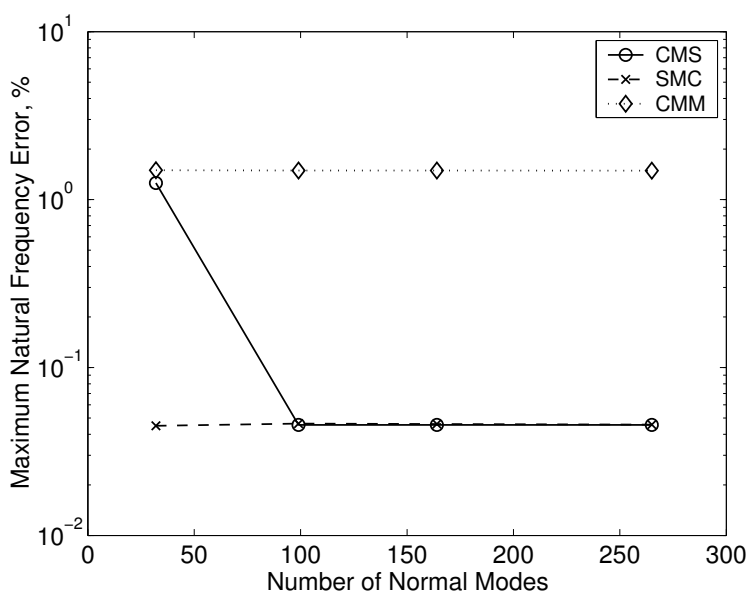

a) 5th mode group

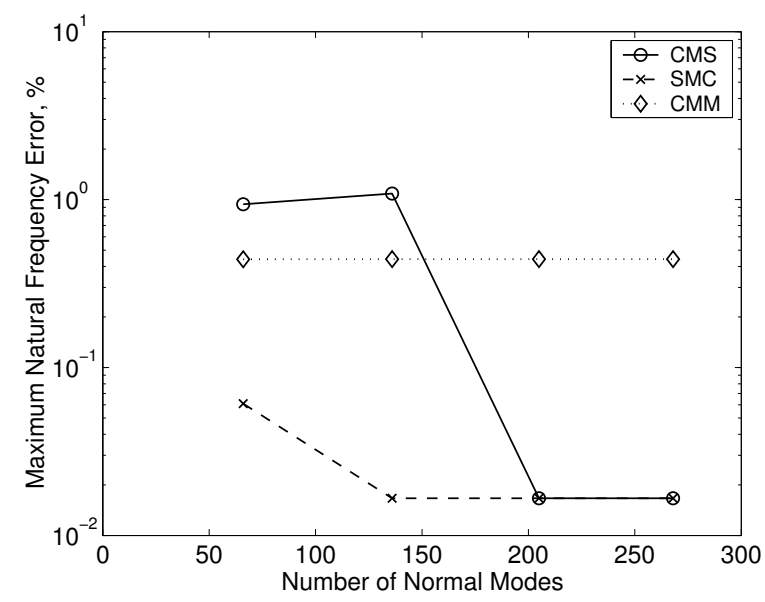

b) 8th and 9th mode groups

Figure 7: Convergence of natural frequency errors for the case of $100 \%$ geometry distortion

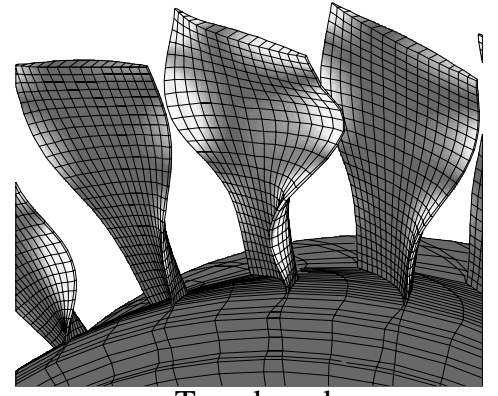

Tuned mode

(33100.23 Hz)

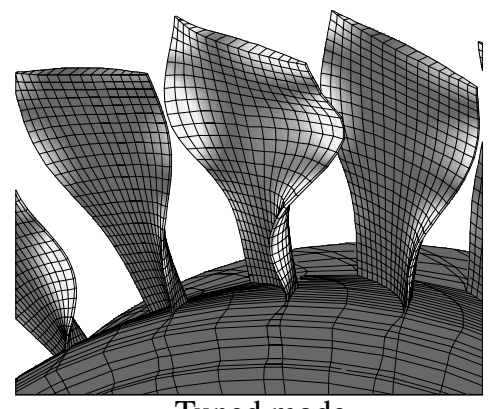

Tuned mode

(33100.23 Hz)

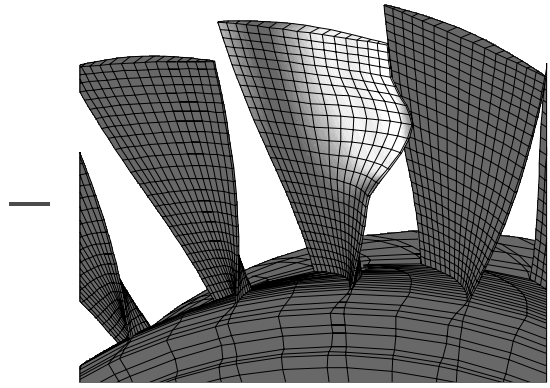

Static mode

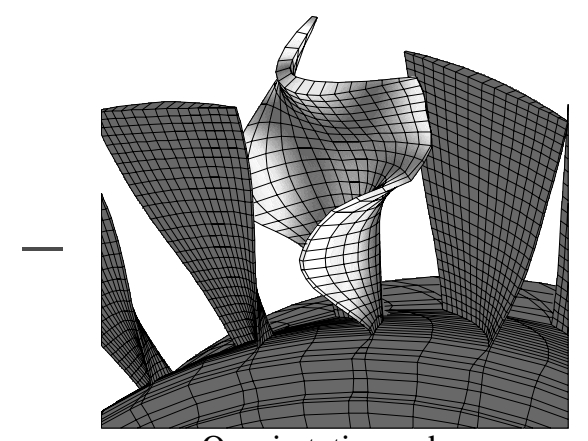

Quasi-static mode (centering frequency: $34000 \mathrm{~Hz}$ )

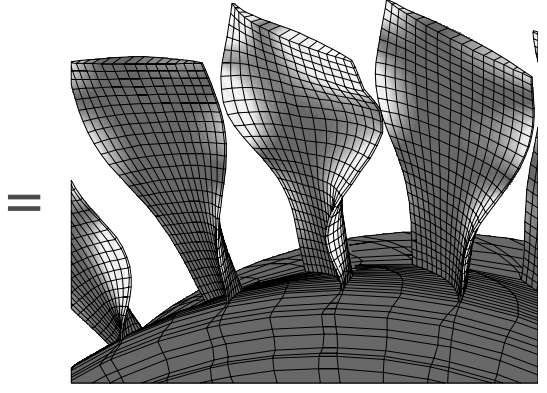

Basis shape

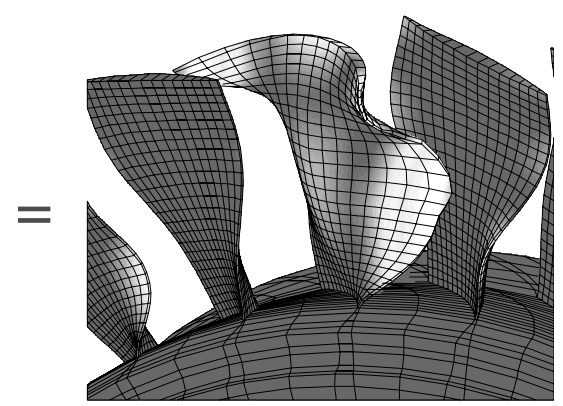

Basis shape

Figure 8: Basis shapes obtained by a static mode and by a quasi-static mode 
Table 1: Convergence of MAC values for the rogue-blade-dominated modes calculated with various ROMs, using the FEM modes as reference

\begin{tabular}{c|c|c|c|c}
\hline Mode group & $\begin{array}{c}\text { No. of normal } \\
\text { modes selected }\end{array}$ & $\begin{array}{c}\text { MAC value } \\
\text { (CMS) }\end{array}$ & $\begin{array}{c}\text { MAC value } \\
\text { (SMC) }\end{array}$ & $\begin{array}{c}\text { MAC value } \\
(\mathrm{CMM})\end{array}$ \\
\hline & 29 & 0.996140 & 0.996114 & 0.998239 \\
1st & 59 & 0.996141 & 0.996131 & 0.998237 \\
group & 90 & 0.996141 & 0.996138 & 0.998237 \\
& 123 & 0.996141 & 0.996138 & 0.998237 \\
\hline & 32 & 0.999975 & 0.999636 & 0.930084 \\
5 th & 99 & 0.999992 & 0.999974 & 0.921637 \\
group & 164 & 0.999993 & 0.999987 & 0.921462 \\
& 265 & 0.999994 & 0.999992 & 0.921296 \\
\hline & 66 & 0.974233 & 0.994455 & 0.000723 \\
8th & 136 & 0.993267 & 0.999569 & 0.000688 \\
group & 205 & 0.999217 & 0.999828 & 0.000685 \\
& 268 & 0.999980 & 0.999889 & 0.000680 \\
\hline & 66 & 0.999893 & 0.999854 & 0.355521 \\
9th & 136 & 0.999946 & 0.999943 & 0.356131 \\
group & 205 & 0.999958 & 0.999945 & 0.356410 \\
& 268 & 0.999957 & 0.999951 & 0.356573 \\
\hline
\end{tabular}

FEM. For the mode in the 1st mode group, all three methods show good results. For the 5th mode group, the CMM results are worse than the others. For the 8th and 9th mode groups, the tested modes obtained by the CMM method are completely different from those by the FEM, even though the natural frequency errors were not so bad, as shown in Fig. 7. This is because the mode shapes of a mistuned system with large mistuning cannot be captured properly with a basis of tuned-system normal modes.

For the free response results, the CMS and SMC methods showed a similar degree of accuracy. However, it should be noted that the SMC method shows good accuracy for a much smaller ROM size compared to the CMS method.

\section{Forced Response Results}

For the forced response results, the SMC and CMM methods were considered to compare the performance of a large-mistuning and a small-mistuning ROM. Due to the distorted geometry of the rogue blade, aerodynamic external forces may not be the same as those for a bladed disk with nominal geometry. However, for validation purposes, pure engine order 2 and 5 excitations were considered, and two frequency regions corresponding to the 5 th (19-22 $\mathrm{kHz}$ ), and the 8th and 9th $(32-36 \mathrm{kHz})$ mode groups were investigated. The applied forces were unit loads normal to the blade surface at the tip of each blade. The degree of distortion for the rogue blade was $100 \%$.

Figures 9 and 10 show envelopes of maximum forced response from the ROMs and the FEM. Euclidean displacement norms for every blade were calculated, and the maximum norms were found at every excitation frequency. The 99 tuned-system normal modes in $14-26 \mathrm{kHz}$ were used for the 5th mode group, and the results are plotted in Fig. 9. The 136 modes in 26-43 kHz were used for the for the 8th and 9th mode groups, and the results are depicted in Fig. 10. The results by the SMC method match well with those by the FEM, while the results by the CMM method show big differences for both the 5th and the 8th and 9th mode groups. As can be seen, the difference occurs around the natural frequencies of the rogue-blade-dominated modes $(19.6 \mathrm{kHz}, 32.9 \mathrm{kHz}$, and $34.3 \mathrm{kHz})$. This is because the CMM models cannot capture the rogue-blade-dominated modes. Note that the CMM method yields poor results, even when the MAC value between the 5th cantilevered-blade modes of a tuned and a rogue blades is around 0.9.

Another case was considered with higher MAC values for tuned and rogue cantilevered-blade modes. The same model and external forcing were used, except that the geometry deviation of the rogue blade was $10 \%$. The excitation frequency range was 32-36 kHz. The MAC value was 0.9922 for the 8th cantilevered-blade mode and 0.9916 for the 9th mode (see Fig 3b) ). The forced response results are shown in Fig. 11. Although the mode shapes of the cantilevered-blade modes of the rogue blade are much closer to those of a tuned blade than for the case of $100 \%$ geometry deviation, there are still significant differences between the FEM and CMM forced response results. This indicates that the performance of the CMM method may be very sensitive to geometric mistuning.

12

American Institute of Aeronautics and Astronautics 


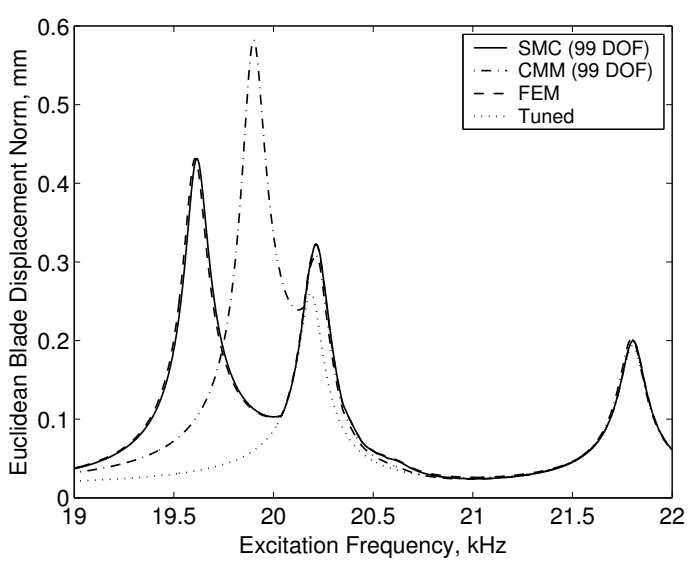

a) Engine order 2 excitation

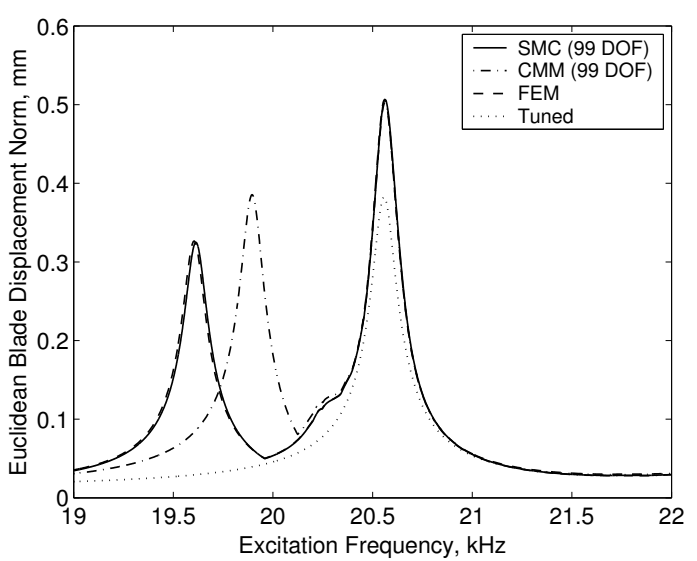

b) Engine order 5 excitation

Figure 9: Forced response in the range $19-21 \mathrm{kHz}$ for $100 \%$ geometry distortion

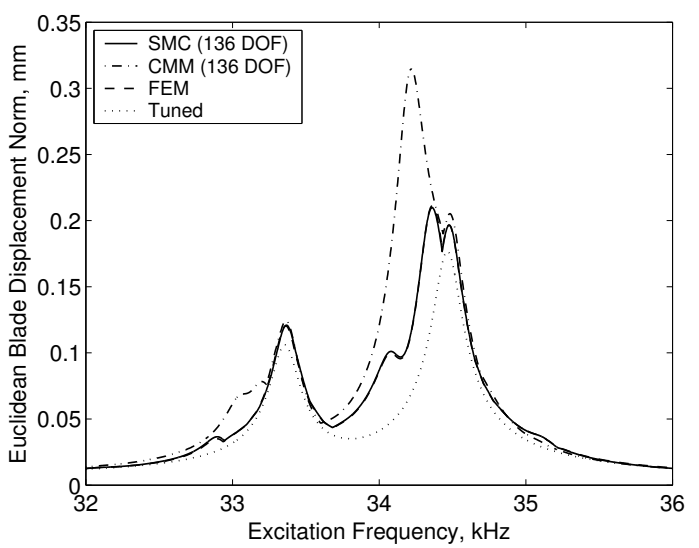

a) Engine order 2 excitation

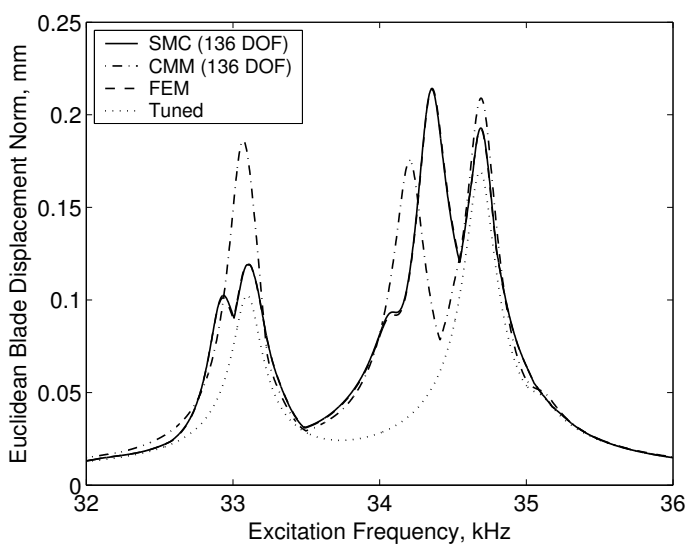

b) Engine order 5 excitation

Figure 10: Forced response in the range $32-36 \mathrm{kHz}$ for $100 \%$ geometry distortion

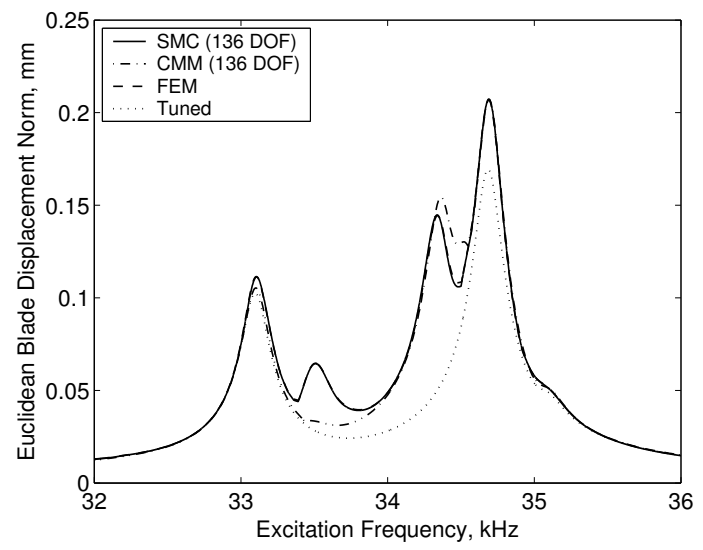

Figure 11: Forced response to engine order 5 excitation in the range 32-36 kHz for 10\% geometry distortion 


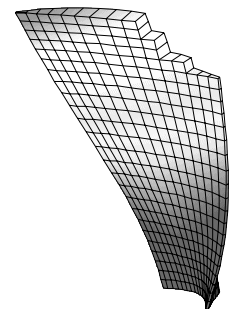

1 st mode $(2318.9 \mathrm{~Hz})$

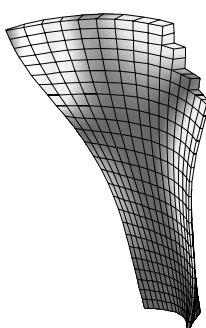

5 th mode

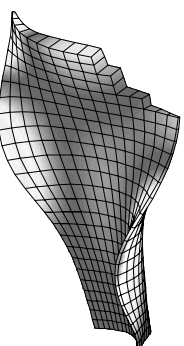

8th mode

$(34141.5 \mathrm{~Hz})$

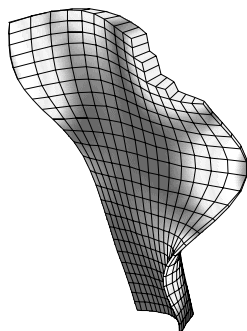

9th mode $(39973.9 \mathrm{~Hz})$

Figure 12: Mode shapes of a fractured blade

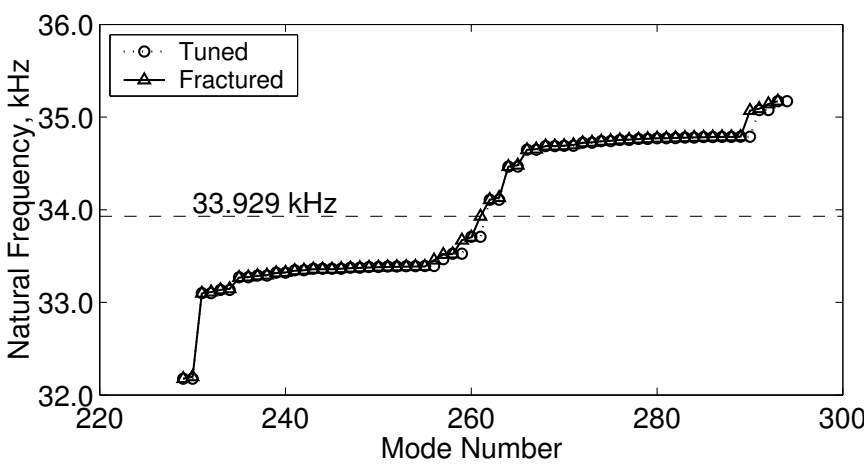

a) Natural frequencies

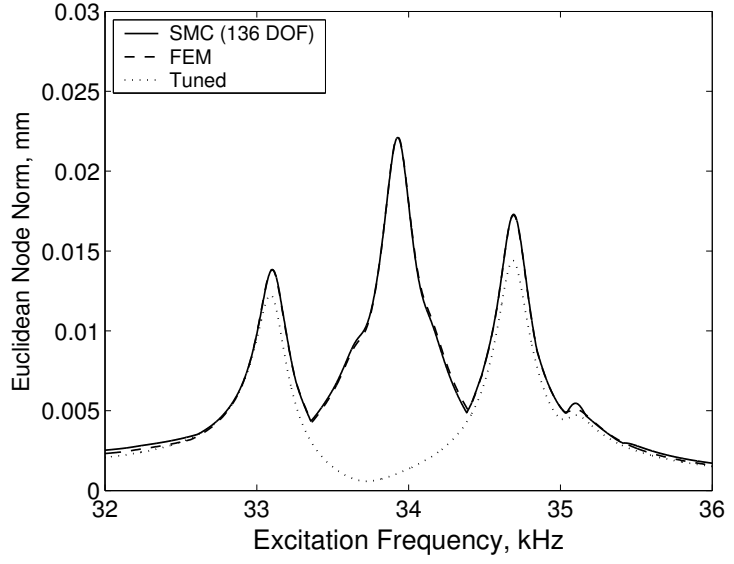

b) Forced response

Figure 13: Free and forced response results for a bladed disk with a fractured blade

\section{Application to a System With a Fractured Blade}

So far, the number of finite element DOF of a tuned system and that of a mistuned system have been assumed to be the same. However, the SMC method can also be used for cases in which some DOF are removed. As an example, a bladed disk with a fractured blade, which is represented by removing some elements from the blade FEM, is discussed in this section.

The same nominal rotor model as used in section IV is employed again. A fractured blade and its mode shapes obtained by fixing its root are shown in Fig. 12. Although finer meshes near the fractured area are required to obtain more accurate results, the original meshes were maintained, since stress concentration is not considered in this study.

Figure 13a) shows the natural frequencies of a bladed disk with the fractured blade for the frequency range 32-36 $\mathrm{kHz}$. A line is shown for $33.929 \mathrm{kHz}$, which is a natural frequency of a fractured-blade-dominated mode. Note that there are 65 mistuned-system normal modes in this frequency range, but there are 66 tuned-system normal modes.

In order to derive the reduced matrices, first, the degrees of freedom in the bladed disk model are sorted into three groups: the removed DOF due to fracture (denoted by $\beta$ ), the DOF at boundaries between the removed part and the remaining part (denoted by $\Gamma$ ), and the interior DOF of the remaining part (denoted by $\alpha$ ). Note that mass and stiffness terms corresponding to $\alpha$ remains unchanged, but the terms corresponding to $\Gamma$ change, when the $\beta$ part is removed. Also, note that there is no mass or stiffness coupling terms between the $\alpha$ part and the $\beta$ part. Therefore, the mass and 
stiffness matrices of the tuned system and the fractured-blade system can be written as follows:

$$
\begin{array}{rlrl}
\boldsymbol{M}^{S}= & {\left[\begin{array}{ccc}
\boldsymbol{M}_{\alpha \alpha} & \boldsymbol{M}_{\alpha \Gamma} & \mathbf{0} \\
\boldsymbol{M}_{\Gamma \alpha} & \boldsymbol{M}_{\Gamma \Gamma} & \boldsymbol{M}_{\Gamma \beta} \\
\mathbf{0} & \boldsymbol{M}_{\beta \Gamma} & \boldsymbol{M}_{\beta \beta}
\end{array}\right],} & \boldsymbol{K}^{S}=\left[\begin{array}{ccc}
\boldsymbol{K}_{\alpha \alpha} & \boldsymbol{K}_{\alpha \Gamma} & \mathbf{0} \\
\boldsymbol{K}_{\Gamma \alpha} & \boldsymbol{K}_{\Gamma \Gamma} & \boldsymbol{K}_{\Gamma \beta} \\
\mathbf{0} & \boldsymbol{K}_{\beta \Gamma} & \boldsymbol{K}_{\beta \beta}
\end{array}\right], \\
\boldsymbol{M}^{m}=\left[\begin{array}{ccc}
\boldsymbol{M}_{\alpha \alpha} & \boldsymbol{M}_{\alpha \Gamma} \\
\boldsymbol{M}_{\Gamma \alpha} & \boldsymbol{M}_{\Gamma \Gamma}+\boldsymbol{M}^{\delta}
\end{array}\right], & \boldsymbol{K}^{m}=\left[\begin{array}{ll}
\boldsymbol{K}_{\alpha \alpha} & \boldsymbol{K}_{\alpha \Gamma} \\
\boldsymbol{K}_{\Gamma \alpha} & \boldsymbol{K}_{\Gamma \Gamma}+\boldsymbol{K}^{\delta}
\end{array}\right],
\end{array}
$$

Then, the required external forces when the motions of a fractured bladed disk are those of tuned-system modes can be computed as follows:

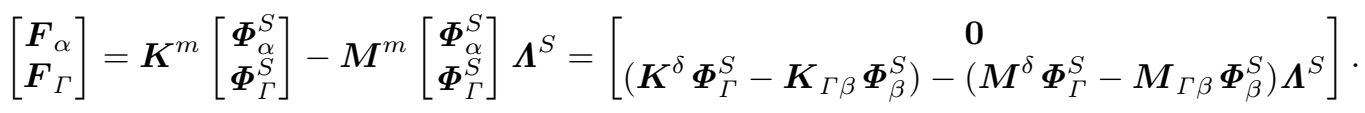

Note that $\boldsymbol{M}^{m}$ and $\boldsymbol{K}^{m}$ are multiplied by only $\alpha$ and $\Gamma$ parts of tuned-system modes, but the $\beta$ part appears in the resulting expression.

Now, as mentioned in section III, static (or quasi-static) modes can be computed in two ways: directly applying $\boldsymbol{F}_{\Gamma}$ to a mistuned system, or applying equivalent forces $\left(\boldsymbol{G}_{\Gamma}\right.$ or $\left.\boldsymbol{P}_{\Gamma}\right)$ to a tuned system. However, since the number of DOF changes in this section, the expression for $\boldsymbol{F}_{\Gamma}$ (Eq. (16)) is different from Eq. (5) so that the expression for equivalent forces should also be different from Eq. (7). It should be noted that the $\beta$ part of the static modes obtained from a tuned system is not used. Therefore, any equivalent forces that can induce the same static deflection in only $\alpha$ and $\Gamma$ parts as those induced by $\boldsymbol{F}_{\Gamma}$ from Eq. (16) can be used. Since there is no coupling between $\alpha$ and $\beta$ parts, any static deflection in $\alpha$ and $\Gamma$ parts of a tuned system can be induced by only the forces on $\alpha$ and $\Gamma$ parts. Furthermore, since $\boldsymbol{F}_{\alpha}$ is $\mathbf{0}, \boldsymbol{G}_{\alpha}$ is also $\mathbf{0}$. That is, any necessary static deflections can be computed by using the quasi-static or static attachment modes corresponding to the DOF in the $\Gamma$ part. So, using quasi-static modes, $\boldsymbol{G}_{\Gamma}$ can be computed from the following equation.

$$
\left(\left[\begin{array}{cc}
\boldsymbol{K}_{\alpha \alpha} & \boldsymbol{K}_{\alpha \Gamma} \\
\boldsymbol{K}_{\Gamma \alpha} & \boldsymbol{K}_{\Gamma \Gamma}+\boldsymbol{K}^{\delta}
\end{array}\right]-\omega_{c}^{2}\left[\begin{array}{cc}
\boldsymbol{M}_{\alpha \alpha} & \boldsymbol{M}_{\alpha \Gamma} \\
\boldsymbol{M}_{\Gamma \alpha} & \boldsymbol{M}_{\Gamma \Gamma}+\boldsymbol{M}^{\delta}
\end{array}\right]\right)\left[\begin{array}{l}
\boldsymbol{\Psi}_{\alpha}^{S, Q} \boldsymbol{G}_{\Gamma} \\
\boldsymbol{\Psi}_{\Gamma}^{S, Q} \boldsymbol{G}_{\Gamma}
\end{array}\right]=\left[\begin{array}{c}
\mathbf{0} \\
\boldsymbol{F}_{\Gamma}
\end{array}\right],
$$

where $\Psi^{S, Q}$ is a set of quasi-static attachment modes corresponding to the DOF in the $\Gamma$ part. Since

$$
\begin{array}{r}
\left(\boldsymbol{K}_{\alpha \alpha}-\omega_{c}^{2} \boldsymbol{M}_{\alpha \alpha}\right) \boldsymbol{\Psi}_{\alpha}^{S, Q}+\left(\boldsymbol{K}_{\alpha \Gamma}-\omega_{c}^{2} \boldsymbol{M}_{\alpha \Gamma}\right) \boldsymbol{\Psi}_{\Gamma}^{S, Q}=\mathbf{0} \\
\left(\boldsymbol{K}_{\Gamma \alpha}-\omega_{c}^{2} \boldsymbol{M}_{\Gamma \alpha}\right) \boldsymbol{\Psi}_{\alpha}^{S, Q}+\left(\boldsymbol{K}_{\Gamma \Gamma}-\omega_{c}^{2} \boldsymbol{M}_{\Gamma \Gamma}\right) \boldsymbol{\Psi}_{\Gamma}^{S, Q}+\left(\boldsymbol{K}_{\Gamma \beta}-\omega_{c}^{2} \boldsymbol{M}_{\Gamma \beta}\right) \boldsymbol{\Psi}_{\beta}^{S, Q}=\boldsymbol{I},
\end{array}
$$

the equivalent force $\boldsymbol{G}_{\Gamma}$ becomes

$$
\boldsymbol{G}_{\Gamma}=\left(\boldsymbol{I}+\left(\boldsymbol{K}^{\delta}-\omega_{c}^{2} \boldsymbol{M}^{\delta}\right) \boldsymbol{\Psi}_{\Gamma}^{S, Q}-\left(\boldsymbol{K}_{\Gamma \beta}-\omega_{c}^{2} \boldsymbol{M}_{\Gamma \beta}\right) \boldsymbol{\Psi}_{\beta}^{S, Q}\right)^{-1} \boldsymbol{F}_{\Gamma}
$$

Using the quasi-static modes obtained in this way, the reduced mass and stiffness matrices can be obtained in the same way as in section III, except that only the mode shapes at the DOF corresponding to the $\alpha$ and $\Gamma$ parts are used.

Since the number of DOF in the $\Gamma$ part of the fractured blade is 72 , the 72 attachment modes were employed to compute the necessary static modes. The centering frequency was chosen to be $34 \mathrm{kHz}$. The tuned-system normal modes selected were 136 modes in $26-43 \mathrm{kHz}$. Figure 13b) shows the forced response results by the obtained ROM and by the FEM. The same unit forces were applied as in section IV, and engine order 5 excitation was considered. For the forced response amplitudes, the maximum value was taken at every frequency among 29 Euclidean displacement norms for nodes located at every blade tip end. As can be seen, the results by the ROM and the FEM match very well. Note that the peak around $34 \mathrm{kHz}$ is due to the fractured-blade-dominated mode at $33.929 \mathrm{kHz}$. 


\section{Application to a System Subject to Design Changes}

When an engineer modifies a design of a bladed disk for a certain purpose, new full FEMs or single-sector FEMs need to be analyzed again to check the effect of the modification. However, using the SMC method, the dynamic responses of the modified system can be easily obtained without repeated FEM analyses by considering mass and stiffness variation due to design change as mistuning.

The change in mass and stiffness matrices due to design change is considered as mistuning that exists in all the sectors. Here, note that a modified bladed disk still features cyclic symmetry, and that a modified-system mode of harmonic $h$ is described by only original-system modes of harmonic $h$. Therefore, the SMC method can be formulated for a certain harmonic $h$, and the problem size is more reduced. For example, if $n$ blade-dominated mode groups of an original system are selected in building a SMC model, the size of reduced mass and stiffness matrices will be about $n \times n$ for a single harmonic, or $2 n \times 2 n$ for a double harmonic, not $\left(n \times N_{b}\right) \times\left(n \times N_{b}\right)$, where $N_{b}$ is the number of blades. Of course, in order to obtain modes throughout all the harmonics, one should solve about $N_{b} / 2$ problems.

Cyclic symmetry is a useful tool in the analysis of cyclic structures, such as bladed disks. Many researchers have used cyclic symmetry to analyze a whole structure based on a single-sector FEM. ${ }^{10,11,22-24}$ Note that in Bladh et al.'s work, ${ }^{11}$ the definition and application of the real Fourier matrix and a pseudo-block-diagonal matrix, which are used in this section, are well explained.

Reduced matrices are built by projecting mass and stiffness matrices in physical coordinates to a basis (here, a selected set of system normal modes compensated by static modes). This projection can be performed for each sector separately. In order to use cyclic symmetry, all the DOF in a FEM need to be arranged sector by sector, and the DOF in boundaries between two sectors need to appear redundantly in the two sectors. The mass and stiffness matrices of a system can be represented by single-sector mass and stiffness matrices, and the modes in physical (cylindrical) coordinates can be represented with the modes in cyclic coordinates and the real Fourier matrix $(\boldsymbol{F})$ as follows:

$$
\begin{aligned}
\boldsymbol{M}^{S} & =\boldsymbol{I} \otimes \boldsymbol{m}^{S} \\
\boldsymbol{K}^{S} & =\boldsymbol{I} \otimes \boldsymbol{k}^{S} \\
\boldsymbol{\Phi}^{S} & =(\boldsymbol{F} \otimes \boldsymbol{I}) \widetilde{\boldsymbol{\Phi}}^{S} \\
\boldsymbol{\Psi}^{S, Q} & =(\boldsymbol{F} \otimes \boldsymbol{I}) \widetilde{\boldsymbol{\Psi}}^{S, Q}\left(\boldsymbol{F}^{T} \otimes \boldsymbol{I}\right),
\end{aligned}
$$

where $\otimes$ denotes the Kronecker product. Here, $\boldsymbol{m}^{S}$ and $\boldsymbol{k}^{S}$ are mass and stiffness matrices of a single-sector model, and $\sim$ denotes the modes in cyclic coordinates, which is a pseudo-block-diagonal matrix. A pseudo-block-diagonal matrix has $\left(N_{b}+1\right) / 2$ diagonal blocks, if $N$ is odd, or $N_{b} / 2$ blocks, if $N$ is even. The column size of a block corresponding to harmonic $h$ depends on the number of selected modes corresponding to harmonic $h$, and can be different from each other. And, the row size of a block corresponding to a double harmonic is twice the row size of a block corresponding to a single harmonic. Note that $\boldsymbol{I} \otimes \boldsymbol{m}^{S}$ and $\boldsymbol{I} \otimes \boldsymbol{k}^{S}$ are block-diagonal matrices in which each block has the same number of rows and columns.

From Eqs. (5) and (12), the external forces corresponding to the variation of mass and stiffness matrices are represented in cyclic coordinates as follows:

$$
\begin{aligned}
\boldsymbol{P}_{\Gamma}= & {\left[\boldsymbol{I}+\left(\boldsymbol{I} \otimes\left(\boldsymbol{k}^{\delta}-\omega_{c}^{2} \boldsymbol{m}^{\delta}\right)\right)(\boldsymbol{F} \otimes \boldsymbol{I}) \widetilde{\boldsymbol{\Psi}}^{S, Q}\left(\boldsymbol{F}^{T} \otimes \boldsymbol{I}\right)\right]^{-1} } \\
& \times\left[\left(\boldsymbol{I} \otimes \boldsymbol{k}^{\delta}\right)(\boldsymbol{F} \otimes \boldsymbol{I}) \widetilde{\boldsymbol{\Phi}}_{\Gamma}^{S}-\left(\boldsymbol{I} \otimes \boldsymbol{m}^{\delta}\right)(\boldsymbol{F} \otimes \boldsymbol{I}) \widetilde{\boldsymbol{\Phi}}_{\Gamma}^{S} \boldsymbol{\Lambda}^{S}\right] \\
= & (\boldsymbol{F} \otimes \boldsymbol{I})\left[\boldsymbol{I}+\left(\boldsymbol{I} \otimes\left(\boldsymbol{k}^{\delta}-\omega_{c}^{2} \boldsymbol{m}^{\delta}\right)\right) \widetilde{\boldsymbol{\Psi}}^{S, Q}\right]^{-1}\left[\left(\boldsymbol{I} \otimes \boldsymbol{k}^{\delta}\right) \widetilde{\boldsymbol{\Phi}}_{\Gamma}^{S}-\left(\boldsymbol{I} \otimes \boldsymbol{m}^{\delta}\right) \widetilde{\boldsymbol{\Phi}}_{\Gamma}^{S} \boldsymbol{\Lambda}^{S}\right] \\
= & (\boldsymbol{F} \otimes \boldsymbol{I}) \widetilde{\boldsymbol{P}}_{\Gamma},
\end{aligned}
$$

where

$$
\widetilde{\boldsymbol{P}}_{\Gamma}=\left[\boldsymbol{I}+\left(\boldsymbol{I} \otimes\left(\boldsymbol{k}^{\delta}-\omega_{c}^{2} \boldsymbol{m}^{\delta}\right)\right) \widetilde{\boldsymbol{\Psi}}^{S, Q}\right]^{-1}\left[\left(\boldsymbol{I} \otimes \boldsymbol{k}^{\delta}\right) \widetilde{\boldsymbol{\Phi}}_{\Gamma}^{S}-\left(\boldsymbol{I} \otimes \boldsymbol{m}^{\delta}\right) \widetilde{\boldsymbol{\Phi}}_{\Gamma}^{S} \boldsymbol{\Lambda}^{S}\right] .
$$

Here, $\widetilde{\boldsymbol{P}}$ is a pseudo-block-diagonal matrix representing the external force matrix in cyclic coordinates.

Now, by replacing all the matrices in Eq. (13b) with the above matrices defined in cyclic coordinates, the following 
reduced matrices are obtained.

$$
\begin{aligned}
\boldsymbol{\mu}^{s y n}= & \boldsymbol{I}+\widetilde{\boldsymbol{\Phi}}_{\Gamma}^{S^{T}}\left(\boldsymbol{I} \otimes \boldsymbol{m}^{\delta}\right) \widetilde{\boldsymbol{\Phi}}_{\Gamma}^{S}-\left[\left(\boldsymbol{\Lambda}^{S}-\omega_{c}^{2} \boldsymbol{I}\right)^{-1} \widetilde{\boldsymbol{\Phi}}_{\Gamma}^{S^{T}}+\widetilde{\boldsymbol{\Phi}}_{\Gamma}^{S^{T}}\left(\boldsymbol{I} \otimes \boldsymbol{m}^{\delta}\right) \widetilde{\boldsymbol{\Psi}}_{\Gamma}^{S, Q}\right] \widetilde{\boldsymbol{P}}_{\Gamma} \\
& -\widetilde{\boldsymbol{P}}_{\Gamma}^{T}\left[\widetilde{\boldsymbol{\Phi}}_{\Gamma}^{S}\left(\boldsymbol{\Lambda}^{S}-\omega_{c}^{2} \boldsymbol{I}\right)^{-1}+\widetilde{\boldsymbol{\Psi}}_{\Gamma}^{S, Q^{T}}\left(\boldsymbol{I} \otimes \boldsymbol{m}^{\delta}\right) \widetilde{\boldsymbol{\Phi}}_{\Gamma}^{S}\right] \\
& +\widetilde{\boldsymbol{P}}_{\Gamma}^{T}\left(\widetilde{\boldsymbol{\Psi}}^{S, Q^{T}}\left(\boldsymbol{I} \otimes \boldsymbol{m}^{S}\right) \widetilde{\boldsymbol{\Psi}}^{S, Q}+\widetilde{\boldsymbol{\Psi}}_{\Gamma}^{S, Q^{T}}\left(\boldsymbol{I} \otimes \boldsymbol{m}^{\delta}\right) \widetilde{\boldsymbol{\Psi}}_{\Gamma}^{S, Q}\right) \widetilde{\boldsymbol{P}}_{\Gamma} \\
\boldsymbol{\kappa}^{s y n}= & \boldsymbol{\Lambda}^{S}+\widetilde{\boldsymbol{\Phi}}_{\Gamma}^{S^{T}}\left(\boldsymbol{I} \otimes \boldsymbol{k}^{\delta}\right) \widetilde{\boldsymbol{\Phi}}_{\Gamma}^{S}-\left[\boldsymbol{\Lambda}^{S}\left(\boldsymbol{\Lambda}^{S}-\omega_{c}^{2} \boldsymbol{I}\right)^{-1} \widetilde{\boldsymbol{\Phi}}_{\Gamma}^{S^{T}}+\widetilde{\boldsymbol{\Phi}}_{\Gamma}^{S^{T}}\left(\boldsymbol{I} \otimes \boldsymbol{k}^{\delta}\right) \widetilde{\boldsymbol{\Psi}}_{\Gamma}^{S, Q}\right] \widetilde{\boldsymbol{P}}_{\Gamma} \\
& -\widetilde{\boldsymbol{P}}_{\Gamma}^{T}\left[\widetilde{\boldsymbol{\Phi}}_{\Gamma}^{S}\left(\boldsymbol{\Lambda}^{S}-\omega_{c}^{2} \boldsymbol{I}\right)^{-1} \boldsymbol{\Lambda}^{S}+\widetilde{\boldsymbol{\Psi}}_{\Gamma}^{S, Q^{T}}\left(\boldsymbol{I} \otimes \boldsymbol{k}^{\delta}\right) \widetilde{\boldsymbol{\Phi}}_{\Gamma}^{S}\right] \\
& +\widetilde{\boldsymbol{P}}_{\Gamma}^{T}\left(\widetilde{\boldsymbol{\Psi}}_{\Gamma}^{S, Q}+\omega_{c}^{2} \widetilde{\boldsymbol{\Psi}}^{S, Q^{T}}\left(\boldsymbol{I} \otimes \boldsymbol{m}^{S}\right) \widetilde{\boldsymbol{\Psi}}^{S, Q}+\widetilde{\boldsymbol{\Psi}}_{\Gamma}^{S, Q^{T}}\left(\boldsymbol{I} \otimes \boldsymbol{k}^{\delta}\right) \widetilde{\boldsymbol{\Psi}}_{\Gamma}^{S, Q}\right) \widetilde{\boldsymbol{P}}_{\Gamma} .
\end{aligned}
$$

Note that $\boldsymbol{\mu}^{s y n}$ and $\boldsymbol{\kappa}^{s y n}$ are square pseudo-block-diagonal matrices whose sizes are determined by the number of original-system normal modes selected. Therefore, each block in these matrices can be handled separately according to harmonic number as follows:

$$
\begin{aligned}
\widetilde{\boldsymbol{\mu}}_{h}^{s y n}= & \boldsymbol{I}+\widetilde{\boldsymbol{\Phi}}_{\Gamma, h}^{S}{ }^{T}\left(\boldsymbol{I}_{h} \otimes \boldsymbol{m}^{\delta}\right) \widetilde{\boldsymbol{\Phi}}_{\Gamma, h}^{S}-\left[\left(\boldsymbol{\Lambda}_{h}^{S}-\omega_{c}^{2} \boldsymbol{I}\right)^{-1} \widetilde{\boldsymbol{\Phi}}_{\Gamma, h}^{S}{ }^{T}+\widetilde{\boldsymbol{\Phi}}_{\Gamma, h}^{S}{ }^{T}\left(\boldsymbol{I}_{h} \otimes \boldsymbol{m}^{\delta}\right) \widetilde{\boldsymbol{\Psi}}_{\Gamma, h}^{S, Q}\right] \widetilde{\boldsymbol{P}}_{\Gamma, h} \\
& -\widetilde{\boldsymbol{P}}_{\Gamma, h}^{T}\left[\widetilde{\boldsymbol{\Phi}}_{\Gamma, h}^{S}\left(\boldsymbol{\Lambda}_{h}^{S}-\omega_{c}^{2} \boldsymbol{I}\right)^{-1}+\widetilde{\boldsymbol{\Psi}}_{\Gamma, h}^{S, Q^{T}}\left(\boldsymbol{I}_{h} \otimes \boldsymbol{m}^{\delta}\right) \widetilde{\boldsymbol{\Phi}}_{\Gamma, h}^{S}\right] \\
& +\widetilde{\boldsymbol{P}}_{\Gamma, h}^{T}\left(\widetilde{\boldsymbol{\Psi}}_{h}^{S, Q^{T}}\left(\boldsymbol{I}_{h} \otimes \boldsymbol{m}^{S}\right) \widetilde{\boldsymbol{\Psi}}_{h}^{S, Q}+\widetilde{\boldsymbol{\Psi}}_{\Gamma, h}^{S, Q^{T}}\left(\boldsymbol{I}_{h} \otimes \boldsymbol{m}^{\delta}\right) \widetilde{\boldsymbol{\Psi}}_{\Gamma, h}^{S, Q}\right) \widetilde{\boldsymbol{P}}_{\Gamma, h} \\
\widetilde{\boldsymbol{\kappa}}_{h}^{s y n}= & \boldsymbol{\Lambda}_{h}^{S}+\widetilde{\boldsymbol{\Phi}}_{\Gamma, h}^{S}{ }^{T}\left(\boldsymbol{I}_{h} \otimes \boldsymbol{k}^{\delta}\right) \widetilde{\boldsymbol{\Phi}}_{\Gamma, h}^{S}-\left[\boldsymbol{\Lambda}_{h}^{S}\left(\boldsymbol{\Lambda}_{h}^{S}-\omega_{c}^{2} \boldsymbol{I}\right)^{-1} \widetilde{\boldsymbol{\Phi}}_{\Gamma, h}^{S}{ }^{T}+\widetilde{\boldsymbol{\Phi}}_{\Gamma, h}^{S}{ }^{T}\left(\boldsymbol{I}_{h} \otimes \boldsymbol{k}^{\delta}\right) \widetilde{\boldsymbol{\Psi}}_{\Gamma, h}^{S, Q}\right] \widetilde{\boldsymbol{P}}_{\Gamma, h} \\
& -\widetilde{\boldsymbol{P}}_{\Gamma, h}^{T}\left[\widetilde{\boldsymbol{\Phi}}_{\Gamma, h}^{S}\left(\boldsymbol{\Lambda}_{h}^{S}-\omega_{c}^{2} \boldsymbol{I}\right)^{-1} \boldsymbol{\Lambda}_{h}^{S}+\widetilde{\boldsymbol{\Psi}}_{\Gamma, h}^{S, Q^{T}}\left(\boldsymbol{I}_{h} \otimes \boldsymbol{k}^{\delta}\right) \widetilde{\boldsymbol{\Phi}}_{\Gamma, h}^{S}\right] \\
& +\widetilde{\boldsymbol{P}}_{\Gamma, h}^{T}\left(\widetilde{\boldsymbol{\Psi}}_{\Gamma, h}^{S, Q}+\omega_{c}^{2} \widetilde{\boldsymbol{\Psi}}_{h}^{S, Q^{T}}\left(\boldsymbol{I}_{h} \otimes \boldsymbol{m}^{S}\right) \widetilde{\boldsymbol{\Psi}}_{h}^{S, Q}+\widetilde{\boldsymbol{\Psi}}_{\Gamma, h}^{S, Q^{T}}\left(\boldsymbol{I}_{h} \otimes \boldsymbol{k}^{\delta}\right) \widetilde{\boldsymbol{\Psi}}_{\Gamma, h}^{S, Q}\right) \widetilde{\boldsymbol{P}}_{\Gamma, h},
\end{aligned}
$$

where the subscript $h$ denotes a harmonic number, and $\boldsymbol{I}_{h}$ is an identity matrix. Note that $\boldsymbol{I}_{h}, \widetilde{\boldsymbol{\mu}}_{h}^{\text {syn }}$ and $\widetilde{\boldsymbol{\kappa}}_{h}^{\text {syn }}$ are square matrices whose size is determined by the number of selected normal modes corresponding to harmonic $h$. Therefore, the amount of computation for sector design change is smaller than that for the case in which only one blade has mistuning.

As an example, a case in which the thickness of rim changes is discussed. The same nominal rotor model as used in the previous sections was used for this case. As shown in Fig. 14, the thickness of the rim on one side of the rotor was varied by stretching some elements in the radial direction. The ratio of stretch $(r)$ is defined as the ratio of the radial coordinate of a modified node to that of a original node. The range of stretching ratio was 0.980 to 1.015 with increment being 0.001 . Thereby, 36 different designs were obtained. The ratio 0.980 indicates a thicker rim, and 1.015 indicates a thinner rim. This change of rim thickness will affect disk-dominated modes. The change in natural frequency veering characteristics will be attendant, which can cause significant changes in mistuned forced response levels. ${ }^{25-27}$

The 34 modes in the range $26-30 \mathrm{kHz}$ were observed as the thickness of the rim was varied. In order to build a model, 34 normal modes of the original system are selected. For quasi-static modes, $27 \mathrm{kHz}$ was chosen as the centering frequency. So, 2-DOF models were obtained for harmonics 0 , 1, and 4-14; and 4-DOF models were obtained for harmonics 2 and 3. Figure 15a) shows the plot of the maximum natural frequency errors versus the stretching ratio. When the stretching ratio is 1 , the error is zero, because there is no modification. As can be seen, the error level tends to increase as the amount of stretch increases, although the error level is quite small.

The variations of natural frequencies resulting from the 36 thickness variations are depicted in Fig. 15b). As would be expected, the natural frequencies become lower as the thickness decreases. The natural frequencies of bladedominated modes, which are located at harmonics 1 and 4-14, do not change much. The rim thickness changes affect only disk-dominated modes. Figure $15 \mathrm{~b}$ ) also shows the variation of a harmonic 0 mode shape. As the rim becomes thinner, less blade motion and more disk motion is observed in the mode shape.

As shown in this case study, the SMC method can be used to evaluate the effects of geometric design changes. If a design is changed just once, a finite element analysis may be more efficient. The cost of computing normal modes may be cheaper than that for computing static modes and building a ROM. However, if the geometry modification of a 


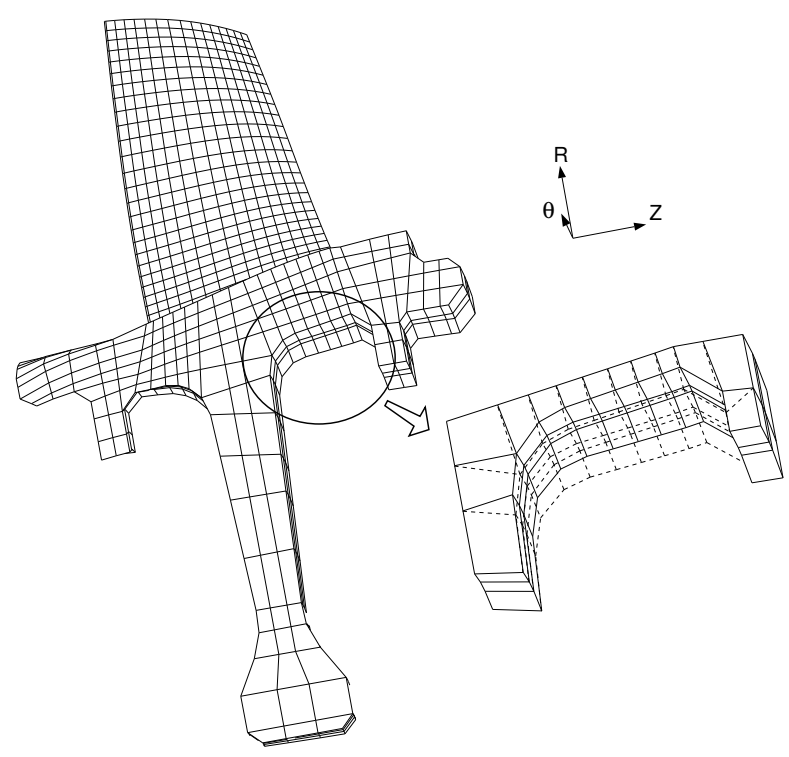

Figure 14: Stretch of rim in radial direction

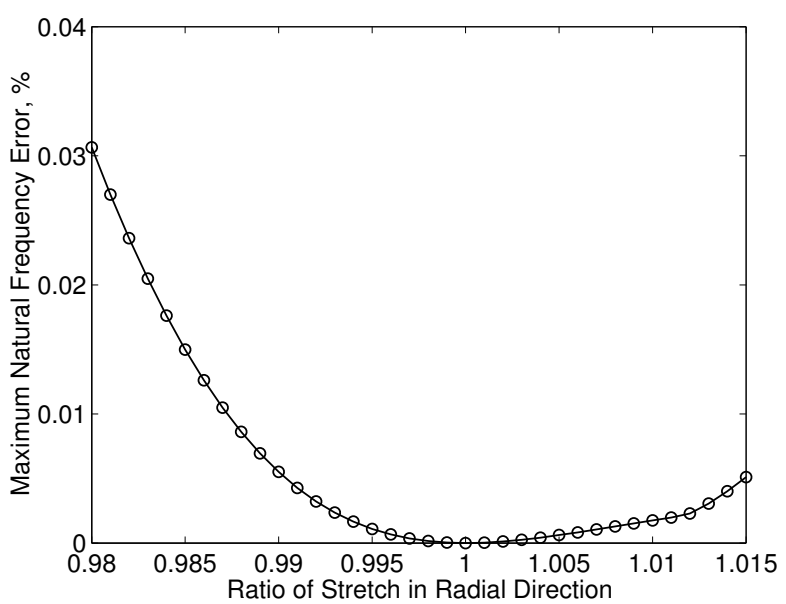

a) Natural frequency errors

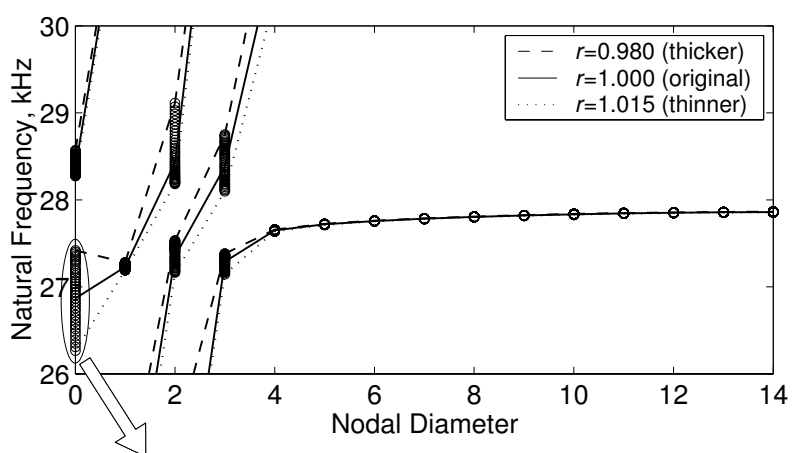

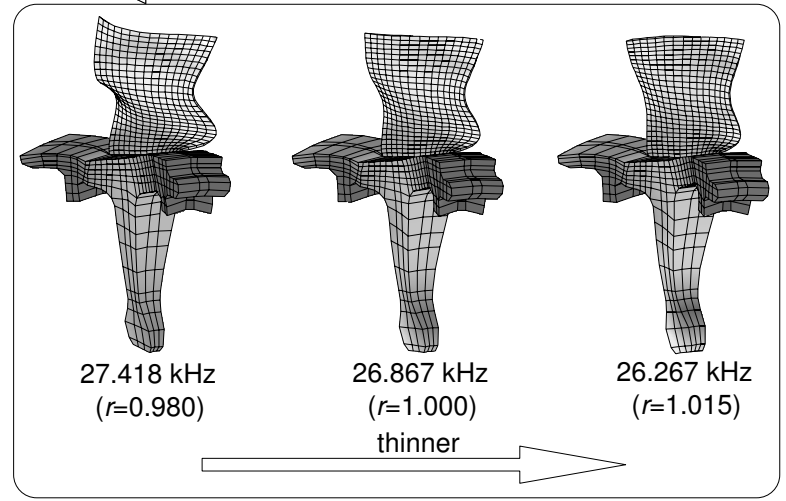

b) Variation of natural frequencies and mode shapes

Figure 15: Effect of rim thickness change on free response 
certain region needs to be repeated for a design sensitivity analysis or a design optimization procedure, then the SMC method will provide improved computational efficiency.

\section{Conclusions}

A new reduced-order modeling technique for bladed disks with large, geometric mistuning has been developed by utilizing the mode-acceleration method. In this technique, by converting the effect of mistuning to that of external forces, static modes used in the mode-acceleration method are computed. In order to improve the convergence and accuracy in a higher frequency range, the original mode-acceleration formulation was modified, and quasi-static modes were used. In the modified formulation, the advantage of quasi-static modes was clearly observed. The developed technique produces a reduced-order model whose size is comparable to that generated by previously developed techniques for small mistuning.

The newly developed technique was compared with three other reduced-order modeling techniques using a testcase model that had a rogue blade whose geometry was severely distorted. The reduced-order model obtained by a component mode synthesis technique reported in the authors' previous work gave good accuracy. However, the size of the model was much larger than those of the other methods. The small-mistuning model using classical modal analysis could not capture mistuned-system normal modes, even when geometry deviation was small. Another smallmistuning model by the CMM method could capture the motion of the mistuned system only when the change in the mode shapes of a cantilevered blade was negligible. As the amount of the mode-shape change increased, the error of the CMM model increased, especially in the estimation of the rogue-blade-dominated modes. This indicates that, even when a mode shape of a blade differs only slightly from that of the nominal blade, a small-mistuning model may not be able to predict the behavior of the bladed disk. In contrast, the results obtained by the newly developed technique showed good agreement with those by the FEM, regardless of the amount of the mode-shape change due to mistuning.

As other applications, a bladed disk with a fractured blade and a bladed disk subject to a geometric design change were examined. The case of a fractured blade, which was represented by removing some elements from the finite element model of the blade, showed that the new technique could be used even for the extreme case of a blade with missing material. Furthermore, it was seen that the new method can be used for the efficient evaluation of the effects of geometric design changes on the system vibration response.

\section{References}

1 Whitehead, D. S., "Effect of Mistuning on the Vibration of Turbomachine Blades Induced by Wakes," Journal Mechanical Engineering Science, Vol. 8, No. 1, 1966, pp. 15-21.

2 Dye, R. C. F. and Henry, T. A., "Vibration Amplitudes of Compressor Blades Resulting From Scatter in Blade Natural Frequencies," ASME Journal of Engineering for Power, Vol. 91, No. 3, 1969, pp. 182-188.

3 Wagner, J. T., "Coupling of Turbomachine Blade Vibrations Through the Rotor," ASME Journal of Engineering for Power, Vol. 89, No. 4, 1967, pp. 502-512.

${ }^{4}$ Ewins, D. J. and Han, Z. S., "Resonant Vibration Levels of a Mistuned Bladed Disk," ASME Journal of Vibration, Acoustics, Stress, and Reliability in Design, Vol. 106, No. 2, 1984, pp. 211-217.

5 El-Bayoumy, L. E. and Srinivasan, A. V., "Influence of Mistuning on Rotor-Blade Vibrations," AIAA Journal, Vol. 13, No. 4, 1975, pp. 460-464.

${ }^{6}$ Griffin, J. H. and Hoosac, T. M., "Model Development and Statistical Investigation of Turbine Blade Mistuning," ASME Journal of Vibration, Acoustics, Stress, and Reliability in Design, Vol. 106, 1984, pp. 204-210.

7 Lin, C.-C. and Mignolet, M. P., "An Adaptive Perturbation Scheme for the Analysis of Mistuned Bladed Disks," ASME Journal of Engineering for Gas Turbines and Power, Vol. 119, No. 1, 1997, pp. 153-160.

8 Wei, S. T. and Pierre, C., "Localization Phenomena in Mistuned Assemblies with Cyclic Symmetry, Part I: Free Vibrations," ASME Journal of Vibration, Acoustics, Stress, and Reliability in Design, Vol. 110, No. 4, 1988, pp. 429438.

9 Óttarsson, G. S. and Pierre, C., "On the Effects of Interblade Coupling on the Statistics of Maximum Forced Response Amplitudes in Mistuned Bladed Disks," AIAA Paper 95-1494, Proceedings of the 36th AIAA/ASME/ASCE/AHS Structures, Structural Dynamics, and Materials Conference, Vol. 5, AIAA, New York, NY, 1995, pp. 3070-3078. 
10 Castanier, M. P., Óttarsson, G., and Pierre, C., “A Reduced-Order Modeling Technique for Mistuned Bladed Disks," Journal of Vibration and Acoustics, Vol. 119, No. 3, 1997, pp. 439-447.

11 Bladh, R., Castanier, M. P., and Pierre, C., "Reduced Order Modeling and Vibration Analysis of Mistuned Bladed Disk Assemblies with Shrouds," ASME Journal of Engineering for Gas Turbines and Power, Vol. 121, No. 3, 1999, pp. 515-522.

12 Bladh, R., Castanier, M. P., and Pierre, C., "Component-Mode-Based Reduced Order Modeling Techniques for Mistuned Bladed Disks-Part I: Theoretical Models," ASME Journal of Engineering for Gas Turbines and Power, Vol. 123, No. 1, 2001, pp. 89-99.

13 Moyroud, F., Fransson, T., and Jacquet-Richardet, G., "A comparison of two finite element reduction techniques for mistuned bladed disks," Journal of Engineering for Gas Turbines and Power, Vol. 124, No. 4, 2002, pp. 942-952.

14 Yang, M.-T. and Griffin, J. H., "A Reduced Order Model of Mistuning Using a Subset of Nominal System Modes," ASME Journal of Engineering for Gas Turbines and Power, Vol. 123, 2001, pp. 893-900.

15 Lim, S., Bladh, R., Castanier, M. P., and Pierre, C., "A Compact, Generalized Component Mode Mistuning Representation for Modeling Bladed Disk Vibration," AIAA Paper 20003-1545, Proceedings of the 44th AIAA/ASME/ASCE/AHS Structures, Structural Dynamics, and Materials Conference, Vol. 2, AIAA, 2003, pp. $1359-1380$.

16 Irretier, H., "Spectral Analysis of Mistuned Bladed Disk Assemblies by Component Mode Synthesis," Vibrations of Bladed Disk Assemblies, ASME, New York, 1983, pp. 115-125.

$17 \mathrm{Gu}, \mathrm{K}$. and Tongue, B. H., "Method to improve the modal convergence for structures with external forcing," ASME Journal of Applied Mechanics, Vol. 54, No. 4, 1987, pp. 904-909.

18 Cai, C. W., Cheung, Y. K., and Chan, H. C., "Mode Localization Phenomena in Nearly Periodic Systems," ASME Journal of Applied Mechanics, Vol. 62, No. 1, 1995, pp. 141-149.

19 Shyu, W.-H., Ma, Z.-D., and Hulbert, G. M., "A New Component Mode Synthesis Method: Quasi-Static Mode Compensation," Finite Elements in Analysis and Design, Vol. 24, 1997, pp. 271-281.

20 Shyu, W.-H., Gu, J., Hulbert, G. M., and Ma, Z.-D., "On the use of multiple quasi-static mode compensation sets for component mode synthesis of complex structures," Finite Elements in Analysis and Design, Vol. 35, 2000, pp. 119-140.

${ }^{21}$ Craig, R. R., “Structural Dynamics, An Introduction to Computer Methods”, Wiley, New York, 1981.

22 Joseph, J. A., "Cyclic Symmetry in MSC/NASTRAN," MSC/NASTRAN Application Manual, chap. 3.2, The MacNeal-Schwendler Corporation, 1981, pp. 10-24.

${ }^{23}$ Elchuri, V., Smith, G. C. C., and Gallo, A. M., "NASTRAN Forced Vibration Analysis of Rotating Cyclic Structures," ASME Journal of Vibration, Acoustics, Stress, and Reliability in Design, Vol. 106, 1984, pp. 224-234.

${ }^{24}$ Hitchings, D. and Singh, M., "Cyclic Symmetry Through Constraint Equations with Application to the Analysis of Steam Turbines," in Bladed Disk Assemblies, Proceedings of the ASME 11th Biennial Conference on Mechanical Vibration and Noise, Boston, Massachusetts, 1987, pp. 113-119.

25 Pierre, C., "Mode Localization and Eigenvalue Loci Veering Phenomena in Disordered Structures," Journal of Sound and Vibration, Vol. 126, No. 3, 1988, pp. 485-502.

${ }^{26}$ Bladh, R., Castanier, M. P., Pierre, C., and Kruse, M. J., "Dynamic Response Predictions for a Mistuned Industrial Turbomachinery Rotor Using Reduced Order Modeling," ASME Journal of Engineering for Gas Turbines and Power, Vol. 124, No. 2, 2002, pp. 311-324.

27 Bladh, R., Castanier, M. P., and Pierre, C., "Effects of Multistage Coupling and Disk Flexibility on Mistuned Bladed Disk Dynamics," ASME Journal of Engineering for Gas Turbines and Power, Vol. 125, No. 1, 2003, pp. 121-130. 